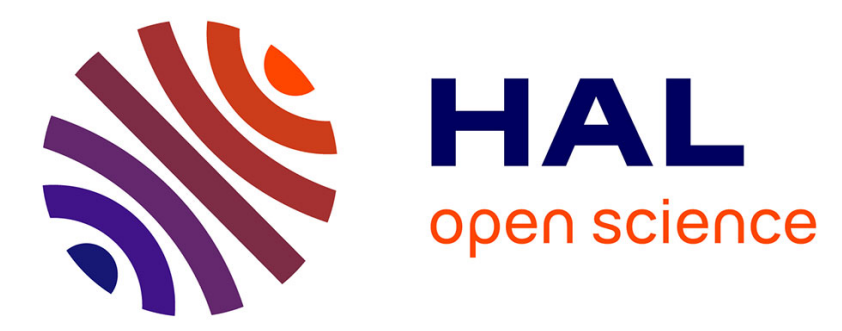

\title{
Réflexions sur l'usage des larnakès et cercueils en Grèce Mycénienne
}

\author{
Laetitia Phialon, Sandrine Farrugio
}

\section{To cite this version:}

Laetitia Phialon, Sandrine Farrugio. Réflexions sur l'usage des larnakès et cercueils en Grèce Mycénienne. Revue archéologique, 2005, 40 (2), pp.227-254. 10.3917/arch.052.0227 . hal-02614947

\section{HAL Id: hal-02614947 \\ https://hal.science/hal-02614947}

Submitted on 3 Jun 2020

HAL is a multi-disciplinary open access archive for the deposit and dissemination of scientific research documents, whether they are published or not. The documents may come from teaching and research institutions in France or abroad, or from public or private research centers.
L'archive ouverte pluridisciplinaire HAL, est destinée au dépôt et à la diffusion de documents scientifiques de niveau recherche, publiés ou non, émanant des établissements d'enseignement et de recherche français ou étrangers, des laboratoires publics ou privés. 


\title{
RÉFLEXIONS SUR L'USAGE DES LARNAKÈS ET CERCUEILS EN GRÈCE MYCÉNIENNE
}

\author{
Laetitia Phialon et Sandrine Farrugio
}

P.U.F. | Revue archéologique

$2005 / 2-n^{\circ} 40$

pages 227 à 254

ISSN 0035-0737

Article disponible en ligne à l'adresse:

http://www.cairn.info/revue-archeologique-2005-2-page-227.htm

Pour citer cet article :

Phialon Laetitia et Farrugio Sandrine , «Réflexions sur l'usage des larnakès et cercueils en Grèce Mycénienne », Revue archéologique, 2005/2 n40, p. 227-254. DOI : 10.3917/arch.052.0227

Distribution électronique Cairn.info pour P.U.F..

(C) P.U.F.. Tous droits réservés pour tous pays.

La reproduction ou représentation de cet article, notamment par photocopie, n'est autorisée que dans les limites des conditions générales d'utilisation du site ou, le cas échéant, des conditions générales de la licence souscrite par votre établissement. Toute autre reproduction ou représentation, en tout ou partie, sous quelque forme et de quelque manière que ce soit, est interdite sauf accord préalable et écrit de l'éditeur, en dehors des cas prévus par la législation en vigueur en France. II est précisé que son stockage dans une base de données est également interdit. 


\title{
RÉFLEXIONS SUR L'USAGE DES LARNAKÈS ET CERCUEILS EN GRÈCE MYCÉNIENNE
}

\author{
par Laetitia Phialon et Sandrine Farrugio
}

\begin{abstract}
Résumé. - Au Bronze Récent, divers types d'inhumations coexistent au sein d'un même cimetière. Dans cet article, on recense onze cimetières en Grèce continentale qui témoignent d'inhumations en larnakès ou en cercueils. Les cimetières de Tanagra ont livré le plus grand nombre de larnakès en terre cuite. Ces dernières remontent à l'Helladique Récent III (1400 av. J.-C.). Par ailleurs, la présence de contenants funéraires en bois dans les cimetières d'Éleusis et de Pérati a pu être mise en évidence par une étude de la position des restes osseux. Le mobilier des tombes mycéniennes renfermant des contenants funéraires ne se distingue pas du mobilier déposé dans les tombes qui en sont dépourvues. Dans les deux cas, on compte principalement des fragments de coupes. Des ossements de chèvres sont également dégagés. Aussi, les rites funéraires incluant repas et boissons devaient être répandus et ne semblent pas dépendre du mode d'inhumation.
\end{abstract}

Mots clés. - Grèce. Grèce continentale. Tanagra. Éleusis. Crète. Nécropole. Inhumation. Mobilier. Mobilier funéraire. Larnax. Cercueil. Tombe. Rite funéraire. Période mycénienne.
About the use of larnakes and coffins in Mycenean Greece.

Abstract. - In the Late Bronze Age different types of burials could be practised in the same cemetery. This article considers eleven cemeteries in continental Greece which contain inhumations in larnakes or coffins. The Tanagra cemeteries yielded most clay larnakes, dating to LH III (1400 BC). Wooden coffins can be deduced for cemeteries at Eleusis and Perati from the position of the skeletons. The offerings in Mycenaean burials are the same whether there are coffins or not. All contain fragments of drinking cups, sometimes also bones of goats. Funeral rites involving feasts and drinking must have been common and did not depend on the type of burial.

Key-words. - Greece. Continental Greece. Tanagra. Eleusis. Crete. Necropolis. Inhumation. Furniture. Funerary furniture. Larnax. Coffin. Tomb. Funerary rite. Mycenean period.

Au Bronze Récent, divers types d'inhumations coexistent au sein d'une même région ou d'un même cimetière ${ }^{1}$. En Grèce continentale, les inhumations en contenants paraissent peu répandues, alors qu'en Crète ce type d'inhumation est fréquemment attesté. À cette période,

1. Cet article résulte de deux recherches menées respectivement par Laetitia Phialon et Sandrine Farrugio. L'une concerne les larnakès en terre cuite, un thème abordé dans un mémoire de licence à l'Université de Genève en 2001, suivi par J.-L. Zimmermann. L'autre se fonde sur le traitement des défunts, sujet développé dans un mémoire de DEA

Rev. ARCH. 2/2005 soutenu à l'Université de Paris I-Panthéon-Sorbonne en 2003 sous la direction de P. Darcque. Cet article n'aurait pu voir le jour sans l'aide et les précieux conseils de H. Procopiou, P. Darcque, G. Touchais et R. Treuil. Nous leur adressons tous nos remerciements. 


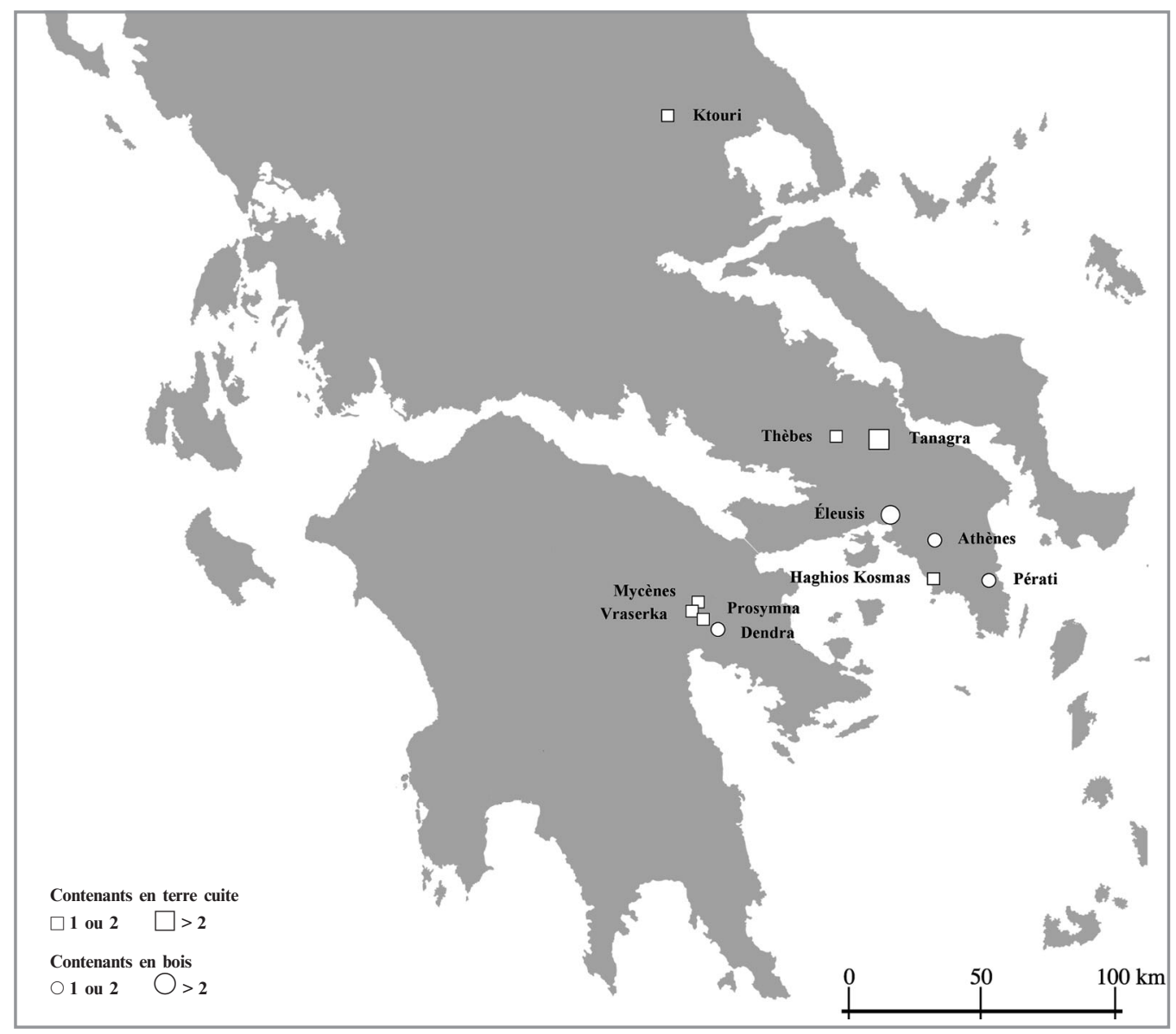

1. Répartition des contenants funéraires en Grèce continentale (L. Phialon).

en Grèce continentale, la déposition des corps directement sur le sol représente le mode d'inhumation le plus pratiqué.

Les analyses menées sur les contenants en terre cuite du Bronze Récent, appelés communément larnakès, sont essentiellement typologiques et stylistiques ${ }^{2}$. Cependant, l'usage de contenants funéraires en Grèce continentale ne s'avère pas systématiquement relevé. Le contexte de découverte reste peu étudié. Jusqu'à présent, seules les traces de bois dans les tombes avaient été jugées révélatrices de la présence d'un contenant périssable. De nouveaux critères, comme la position des os et, dans certains cas, celle du mobilier funéraire, permettent toutefois d'identifier des inhumations en contenants.

2. Voir notamment Vermeule 1965 ; B. Rutkowski, Larnaksy egejskie, Bibliotheca antiqua, 7, Wroclaw, Zak. narodowy im. Ossolinskich, 1966 ; Mavriyannaki 1972. 
Dans cet article, nous examinerons la répartition en Grèce continentale des différents types d'inhumations en contenants funéraires (fig. 1). De plus, nous nous interrogerons sur la concentration de larnakès en terre cuite à Tanagra. Nous tenterons également de discuter les hypothèses émises au sujet des pratiques adoptées lors d'inhumations dans diverses régions.

\section{CONTENANTS ET CONTENUS FUNÉRAIRES}

Les larnakès sont des contenants funéraires dans lesquels sont déposés des adultes ou des enfants. Le défunt est déposé sur le dos, les genoux fléchis au-dessus du fond du contenant ${ }^{3}$. En Grèce continentale, la longueur des plus grandes larnakès ne dépasse pas 1,15 $\mathrm{m}$, tandis qu'en Crète elle atteint parfois $1,50 \mathrm{~m}$. Il arrive que des larnakès contiennent une inhumation secondaire; les os de la première inhumation sont alors repoussés sur les côtés. Parfois, les larnakès ont servi d'ossuaires ${ }^{4}$. L'étude typologique des larnakès en terre cuite et en pierre a permis d'identifier deux types au Bronze Récent : la larnax en forme de coffre et celle en forme de baignoire $^{5}$. Les deux types sont attestés en Crète et en Grèce continentale, principalement dans des tombes à chambre. Dans l'Iliade, $\lambda \alpha \alpha_{\rho} v \alpha \xi$ désigne non seulement un coffre de type domestique, mais aussi une urne à crémation ${ }^{6}$.

Il devait également exister des contenants en matière périssable de dimensions variables. Contrairement aux documents en terre cuite, ces derniers ont laissé peu de traces. Quand le contenant est entièrement décomposé, son identification est déterminée par des effets de délimitation linéaire ${ }^{7}$ au niveau des restes osseux. Dans certains cas, le défunt a été introduit les genoux fortement fléchis. Cette position dénote la présence d'un contenant de petites dimensions proches de celles des larnakès en terre cuite. Dans d'autres cas, le défunt a été déposé en position allongée. Le contenant en bois devait correspondre à une longue caisse rectangulaire ou trapézoïdale faite de planches de bois, dénommée cercueil. L'absence d'étude sur la position originelle des défunts et l'état inégal des publications ne permettent pas toujours de recueillir les indications nécessaires à l'étude du contenu.

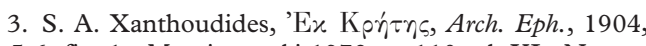
p. 5-6, fig. 1 ; Mavriyannaki 1972, p. 110, pl. XL. Notons que les larnakès en terre cuite apparaissent en Crète, dès le MA III.

4. Mavriyannaki 1972, p. 21 ; Long 1974, p. 20, n. 29.

5. Rutkowski 1968, p. 219, n. 2. L'A. indique que le terme re-wo-te-re-jo en linéaire $\mathrm{B}$ se référant peut-être à une baignoire n'implique aucun usage funéraire. F. Vandenabeele et J.-P. Olivier précisent que re-wo-te-re-jo sur la tablette PY Tn 996 est un adjectif qui qualifie un objet désigné par l'idéogramme représentant une baignoire (transcrit conventionnellement ALV). Cet adjectif peut être traduit par "pour le bain ". C'est le terme a-sa-mi-to qui signifie "baignoire " (KN Ws 8497), voir F. Vandenabeele, J.-P. Olivier, Les idéogrammes archéologiques du linéaire $B$ (Études crétoises, XXIV), Paris, P. Geuthner, 1979, p. 176-180.

6. Iliade, XVIII, 413 ; XXIV, 795.

7. Ensemble de vestiges dont l'organisation forme une limite régulière, plus ou moins continue, dont la cause est due à une paroi qui n'existe plus, voir Duday 1995, p. 47. 


\section{INHUMATIONS EN LARNAKÈS DE TERRE CUITE}

\subsection{Cimetières de Dendron et Géphyra près de Tanagra}

Dans les cimetières de Dendron et Géphyra, situés respectivement à $1000 \mathrm{~m}$ à l'Est et à $400 \mathrm{~m}$ au Sud-Est du village actuel de Tanagra, une soixantaine de larnakès en terre cuite découvertes in situ prouvent que ce type de sépulture était en usage durant l'HR III ${ }^{8}$. La majorité des larnakès provient des fouilles menées sous la direction de Th. G. Spyropoulos de 1968 à 1984, mais près de deux cents tombes avaient déjà fait l'objet de fouilles illicites. Avant le début des investigations, E. T. Vermeule avait identifié, dans divers musées et collections privées, quatorze contenants différents provenant de la région de Tanagra : des larnakès entières ou en fragments'. Il est possible d'ajouter au corpus cinq autres larnakès conservées dans des musées ou des collections privées ${ }^{10}$.

L'inhumation en larnakès concerne au minimum dix-huit tombes de Dendron et dix-sept tombes de Géphyra (tableau 1), sur un total qui atteignait en 1984 cent trente-sept tombes fouillées à Dendron et soixante-treize à Géphyra ${ }^{11}$. Th. G. Spyropoulos rapporte la découverte de cinquante-neuf larnakès en terre cuite dans les deux nécropoles et mentionne la présence de cent trente-six inhumations dégagées sur le sol des chambres et des dromoi, ou dans des fosses creusées dans les tombes. Le nombre de corps déposés directement sur le sol devait être bien plus élevé, car l'état de conservation des ossements s'avérait, dans certains cas, très dégradé.

Parmi les tombes de Dendron et Géphyra qui témoignent d'inhumations en larnakès, la plupart ne renfermaient qu'une seule larnax. Cependant, seize larnakès, qui devaient chacune contenir une inhumation, ont été dégagées dans la tombe 6 de Géphyra ${ }^{12}$. Au contraire, la larnax de la tombe 22 de Géphyra comprenait deux crânes et quelques ossements rassemblés en $\operatorname{tas}^{13}$. Ces deux tombes se démarquent encore par le fait que la tombe $22 \mathrm{n}$ 'a fourni aucune autre trouvaille, tandis que la tombe 6 a livré des vases et des figurines en terre cuite de l'HR III B.

8. Dendron est aussi appelé Gkraba, Géphyra nommé Ledeza, voir Spyropoulos 1969 a, p. 6 ; Spyropoulos 1974, p. 18-19. De plus, le village actuel de Tanagra (ou Vrasi) ne correspond pas à l'antique Tanagra située au Sud-Est $\mathrm{du}$ village actuel de Schimatari - voir D. Roller, Tanagra Survey Project 1985, The Site of Grimadha, $A B S A, 82$, 1987, p. 215, n. 7. Les dates absolues des phases du Bronze Récent en Grèce continentale (ou Helladique Récent) s'établissent de la façon suivante, selon la chronologie basse (éruption de Théra aux environs de 1550 av. J.-C.) : HR I (1550-1500), HR II A (1500-1450), HR II B (14501400), HR III A1 (1400-1370), HR III A2 (13701300), HR III B1 (1300-1225), HR III B2 (1225-1190), HR III C (1190-1050).

9. Vermeule 1965 , p. 123-148. Les larnakès sont numérotées dans l'article du $\mathrm{n}^{\circ} 1$ à 13 , les documents $\mathrm{n}^{\circ} 4$ et $\mathrm{n}^{\circ} 4$ a sont probablement deux larnakès différentes.
10. M. Maass, J. Fabricius éd., Antike Kulturen, Führer durch die Antikensammlungen des Badisches Landesmuseum, Karlsruhe, Badisches Landesmuseum, 1995, p. 61, fig. 56 ; J. Chamay, Sarcophage mycénien, dans J. Dörig éd., Art Antique, Collections privées de Suisse Romande, cat. expo. Genève, 1975, Genève, Éditions archéologiques de l'université, $n^{\circ}$ 96, fig. $96 a-c$; R. Lorandou-Papantoniou, T $\mu \tilde{\mu} \mu \alpha$

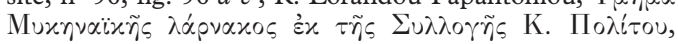
$A A A$, VI, 1973, p. 170 , fig. 1 ; Vermeule 1979 , p. 205, fig. 1 ; P. Demargne, Un sarcophage mycénien de Tanagra, RLouvre, 1988, $\mathrm{n}^{\circ} 1$, p. 13-16.

11. Pour Géphyra, voir Spyropoulos 1971, p. 8 ; pour Dendron, voir Spyropoulos 1984, p. 143-145.

12. Spyropoulos 1969 a, p. 8-9; Spyropoulos 1970 b, p. $185-190$.

13. Spyropoulos 1970 b, p. 190-192. 
Dès le début des fouilles, Th. G. Spyropoulos note que les tombes les plus grandes et les plus riches en mobilier se trouvaient au centre des nécropoles, alors que les tombes modestes étaient dispersées en périphérie ${ }^{14}$. L'abondance de mobilier dans une tombe n'impliquait pas la présence de larnakès, comme en témoigne la tombe 25 de Géphyra dépourvue de larnax, mais comptant des vases et des figurines en terre cuite ${ }^{15}$.

Les deux cimetières mycéniens sont principalement utilisés du début de l'HR III A au début de l'HR III $\mathrm{C}^{16}$. Le mobilier des tombes incluait des centaines de vases et figurines en terre cuite, des perles en pâte de verre. La plupart des vases de Dendron présentent un décor en bandes horizontales caractéristique de l'HR III A2- ${ }^{17}$. Les plus anciens vases remontent toutefois à l'HR III A1, certains peut-être à l'HR II A ${ }^{18}$. À Géphyra, le matériel céramique le plus ancien date de la fin de l'HR III A2, mais la plus grande partie des vases semble être de l'HR III B ${ }^{19}$. D'autres vases datent de l'HR III B-C ${ }^{20}$.

Le mobilier déposé en même temps que les larnakès fournit très peu de repères chronologiques. Dans la nécropole de Dendron, la tombe 3, qui contenait une larnax, est datée par Th. G. Spyropoulos de l'HR III B (fig. 2) ${ }^{21}$. La tombe 31 comprenait une larnax, de nombreux vases de l'HR III A et de l'HR III B ${ }^{22}$. D'après les illustrations, les tombes 97 et 109 contenaient probablement du mobilier de l'HR III B ; la tombe 104, des vases de l'HR III A ${ }^{23}$. Cependant, on ne peut mettre ce mobilier en relation avec une inhumation en larnax. La tombe 115 renfermait une larnax et plusieurs vases, rassemblés en cinq tas et associés à quelques restes osseux ${ }^{24}$. Les plus anciens vases sont datés par P. A. Mountjoy de l'HR II, d'autres remontent sans doute à l'HR III A-B ${ }^{25}$.

D'après les motifs et figures peints, on peut suggérer que les plus anciennes larnakès ornées de motifs végétaux (FM 12.g, FM 15.11, FM 18.63) datent de l'HR III A2. Il est alors probable que la larnax de la tombe 115 date de cette période ${ }^{26}$. Les oiseaux représentés sur d'autres larnakès remontent certainement à l'HR III A2 (voir FM 7.f, 7.4) ${ }^{27}$. La provenance exacte des premières larnakès n'est toutefois pas toujours indiquée ${ }^{28}$.

14. Spyropoulos 1969 a, p. 6-7. Pour d'autres indications topographiques, voir Spyropoulos 1974, p. 18 ; Spyropoulos 1975 , p. 417.

15. Spyropoulos 1969 a, p. 14, pl. $10 \alpha-\beta, 11 \alpha$.

16. Spyropoulos 1971 , p. 10, 14 ; Spyropoulos 1973, p. 20. Les premières tombes dans la partie Ouest de Dendron pourraient même remonter à l'HR II, voir Spyropoulos 1984, p. 143. Le matériel est exposé au Musée archéologique de Thèbes, voir Dimakopoulou et Konsola 1981, p. $80-86$.

17. Spyropoulos 1970 a, p. 32-33. Il s'agit principalement de pyxides, d'alabastres, de jarres à étrier, de coupes, de kyathoi, de cruches, de petites amphores pithoïdes à trois anses, de thélastrons, de kylikès, de skyphoi (ou " deep bowls ") et de kernoi.

18. Mountjoy 1999, p. 643, n. 42 (FS 81, FS 91).

19. Spyropoulos 1971 , p. 10 ; Spyropoulos 1970 b, p. 197. Diverses idoles et figurines en terre cuite, dont des idoles en $\Psi$ et en $\Phi$, des figurines de taureaux, d'oiseaux, de chiens, des tables trépieds et des sièges ont été mis au jour à Géphyra.
20. Spyropoulos 1971, p. 14 .

21. Spyropoulos 1970 a, p. 34 .

22. Spyropoulos 1970 a, p. 31, fig. 1, pl. $42 \alpha, 43 \beta-\gamma$, $46 \beta$.

23. Spyropoulos 1976 , p. 64-65, pl. $32 \alpha-\delta$, pl. $33 \alpha$; Spyropoulos 1977 , p. 28 , fig. $2 \alpha-\beta$, p. 31 , pl. $13 \varepsilon$.

24. Spyropoulos 1979, p. 30, 33-35, 38, pl. 19.

25. Spyropoulos 1979, p. 33-35, pl. $18 \beta-\sigma \tau, 19 \alpha-\varepsilon$; voir Mountjoy 1999, p. 650, fig. 247, $\mathrm{n}^{\circ} 8$ (alabastre), p. 653 , fig. $248, \mathrm{n}^{\circ} 22$ (jarre).

26. Spyropoulos 1979 , p. 30, 33-35, pl. $20 \beta$.

27. Voir aussi Vermeule 1965, p. 130-131, pl. XXVIII $a$; Dimakopoulou et Konsola 1981, p. 83, $\mathrm{n}^{\circ} 11$. Les larnakès des tombes 15 et 32 de Dendron ornées de sphinx doivent aussi remonter à l'HR III A2, voir Spyropoulos 1974, p. 14, pl. $10 \beta, 11 \alpha-\beta$; Spyropoulos 1970 a, p. 35, pl. $48 \beta$.

28. Spyropoulos 1976, p. 68, pl. $33 \varepsilon$; Ergon, 1983, p. 52 , fig. 65 . 
Tableau 1. - Corpus des sépultures témoignant d'inhumations en contenants funéraires dans des cimetières de Grèce continentale*

\begin{tabular}{|c|c|c|c|c|c|c|c|c|}
\hline \multirow{4}{*}{ Sites } & \multicolumn{3}{|c|}{ Tombes rectangulaires construites } & \multicolumn{4}{|c|}{ Tombes à chambre } & \multirow[t]{4}{*}{ Datation } \\
\hline & \multirow[t]{3}{*}{$\begin{array}{c}\text { Dénomi- } \\
\text { nation } \\
\text { des tombes }\end{array}$} & \multirow{2}{*}{\multicolumn{2}{|c|}{$\begin{array}{l}\text { Nombre de sépultures } \\
\text { à l'intérieur des tombes } \\
\text { Contenants en bois }\end{array}$}} & \multirow[t]{3}{*}{$\begin{array}{c}\text { Dénomi- } \\
\text { nation } \\
\text { des tombes }\end{array}$} & \multicolumn{3}{|c|}{$\begin{array}{l}\text { Nombre de sépultures } \\
\text { à l'intérieur des chambres funéraires }\end{array}$} & \\
\hline & & & & & \multicolumn{2}{|c|}{ Contenants en bois } & \multirow{2}{*}{$\begin{array}{c}\text { Contenants } \\
\text { en terre cuite } \\
\text { Larnakès }\end{array}$} & \\
\hline & & Larnakès & Cercueils & & Larnakès & Cercueils & & \\
\hline \multirow[t]{7}{*}{ Éleusis } & Tombe E $\pi 1$ & 2 & & & & & & HM-HR III B \\
\hline & Tombe $\mathrm{H} \pi 5$ & & 1 & & & & & HM-HR II \\
\hline & Tombe $\Theta \pi 4$ & 1 & & & & & & HM-HR II \\
\hline & Tombe $\Theta \pi 5$ & 1 & & & & & & HR III B / C \\
\hline & Tombe $\Lambda \pi 4$ & & 1 & & & & & HR II-III A \\
\hline & Tombe $M \pi 6$ & 2 & & & & & & HR III A-B \\
\hline & Tombe $\mathrm{M} \pi 9$ & 1 & & & & & & HM-HR III B \\
\hline Athènes & & & & Tombe XL & & 2 & & HR III A1 \\
\hline Pérati & & & & Tombe 57 & 1 & & & HR III C \\
\hline Dendra & & & & Tombe 8 & & 1 & & HR II-III \\
\hline Mycènes & & & & Tombe 502 & & & 1 & HR III \\
\hline Prosymna & & & & Tombe 17 & & & 1 & HR III A2 \\
\hline Vraserka & & & & Tombe 1 & & & 1 & HR III A-B \\
\hline \multirow[t]{16}{*}{ Tanagra, D. } & & & & Tombe 3 & & & 1 & HR III B \\
\hline & & & & Tombe 15 & & & 1 & HR (III) \\
\hline & & & & Tombe 16 & & & 1 & HR (III) \\
\hline & & & & Tombe 24 & & & 1 & HR (III) \\
\hline & & & & Tombe 31 & & & 1 & HR III A-B \\
\hline & & & & Tombe 32 & & & 2 & HR (III) \\
\hline & & & & Tombe 36 & & & 1 & HR (III) \\
\hline & & & & Tombe 47 & & & 1 & HR (III) \\
\hline & & & & Tombe 59 & & & 1 & HR (III) \\
\hline & & & & Tombe 69 & & & $>1$ & HR (III) \\
\hline & & & & Tombe 71 & & & 1 & HR (III) \\
\hline & & & & Tombe 73 & & & $>1$ & HR (III) \\
\hline & & & & Tombe 84 & & & 1 & HR (III) \\
\hline & & & & Tombe 97 & & & 1 & HR III B \\
\hline & & & & Tombe 104 & & & 1 & HR III A \\
\hline & & & & (T. 107-111) & & & 1 & HR (III) \\
\hline
\end{tabular}

^ Les datations des tombes sont proposées par les fouilleurs. Pour les tombes de Tanagra, les rapports de fouilles de Th. G. Spyropoulos sont publiés dans les Praktika et dans deux $A A A$. Des références bibliographiques supplémentaires sont mentionnées ci-dessous, lorsque des inhumations en larnakès ne sont pas décrites dans les Praktika et les $A A A$. 
Tableau 1. - (Suite et fin)

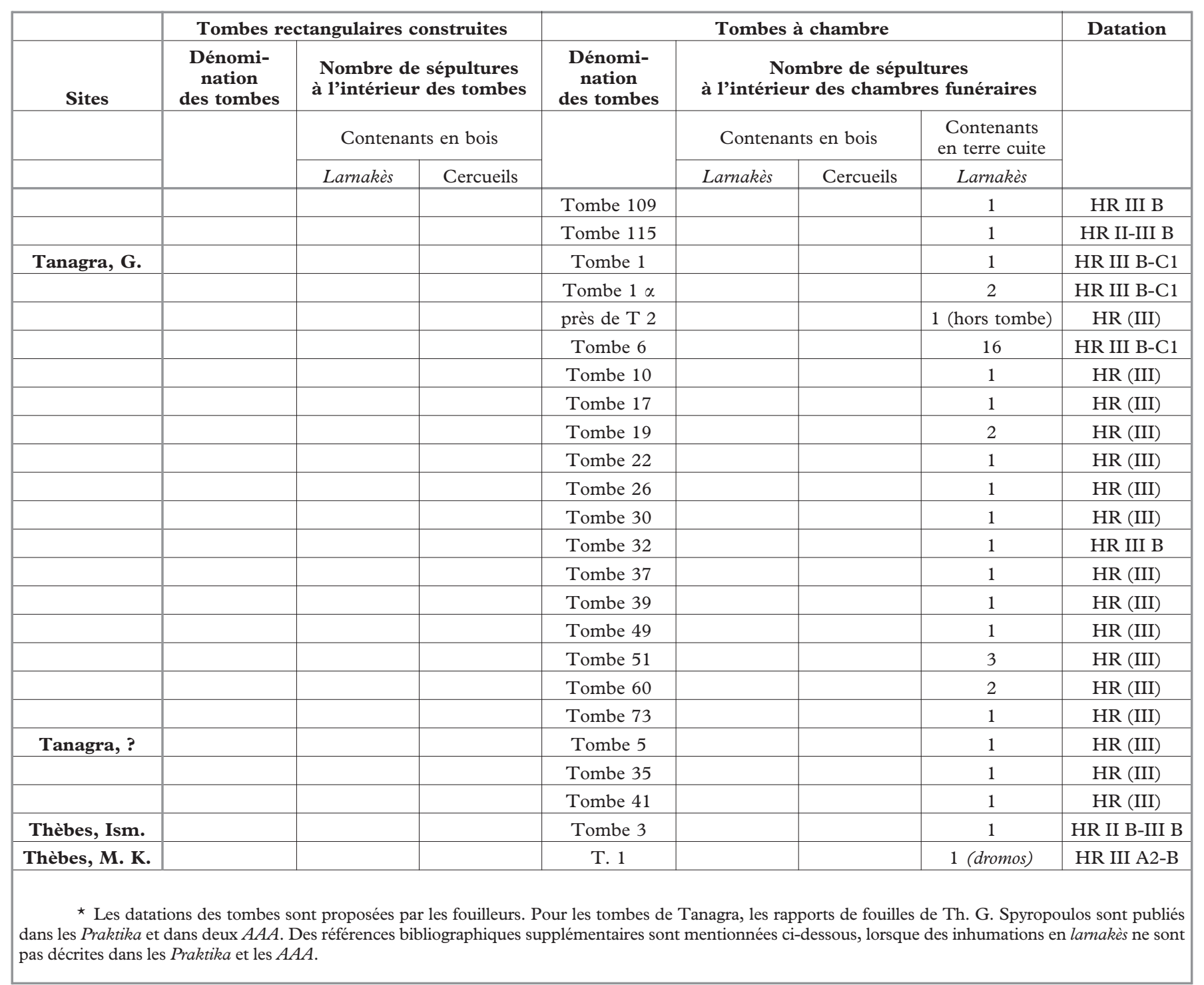


2. Dendron, tombe 3 : scène figurée de la larnax. D'après Spyropoulos 1970 a, pl. $48 \alpha$.

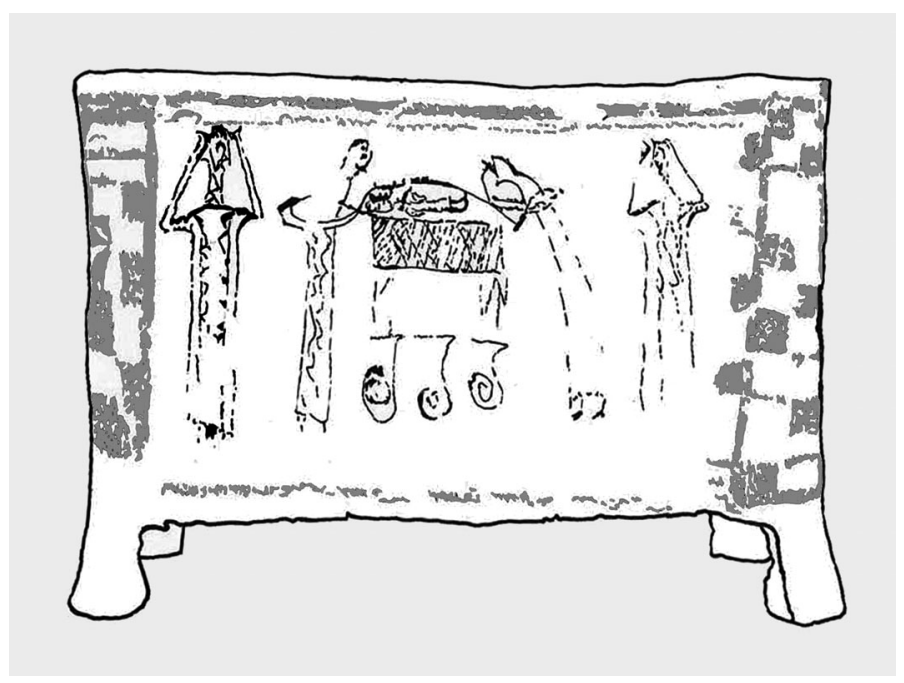

3. Géphyra, tombe 6 : scène figurée d'une larnax. D'après Spyropoulos $1972 b$, pl. 208 a.

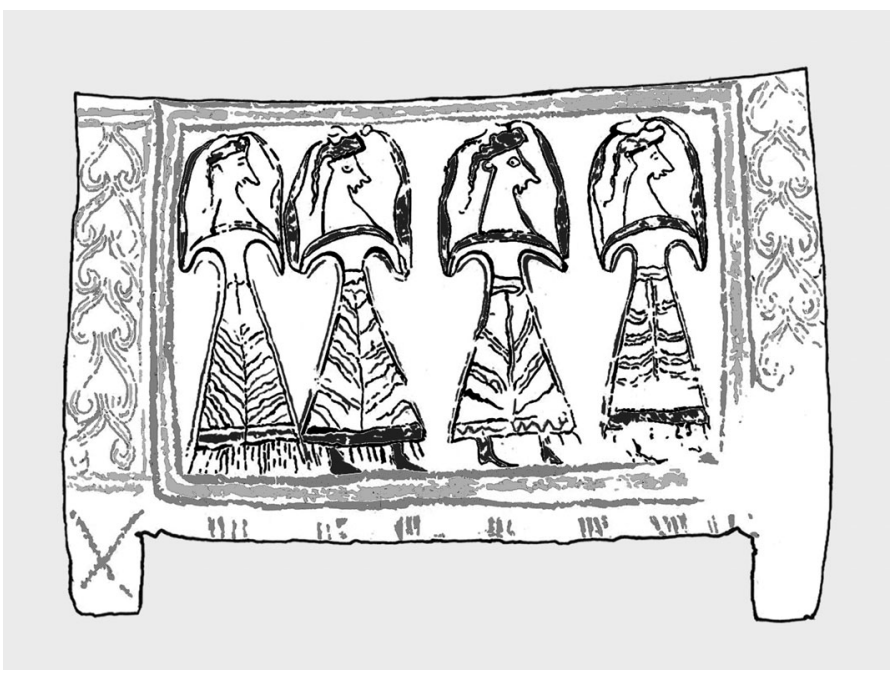

Dans la nécropole de Géphyra, la tombe 6 qui contenait seize larnakès peut être datée par son mobilier de l'HR III B2-C ${ }^{29}$. Le décor des larnakès s'avère de style très proche (fig. 3), les figures humaines arrangées en frise peuvent être comparées aux figures du Vase des Guerriers daté du milieu de l'HR III C $\mathrm{C}^{30}$. La plupart des vases de la tombe $1 \alpha$ sont aussi datés de l'HR III B2-C, mais P. A. Mountjoy date le skyphos trouvé dans la larnax de l'HR III A2 ${ }^{31}$. Le

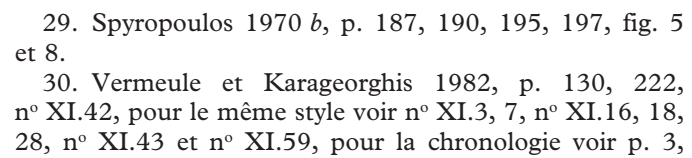

29. Spyropoulos 1970 b, p. 187, 190, 195, 197, fig. 5 et 8 .

30. Vermeule et Karageorghis 1982, p. 130, 222, $\mathrm{n}^{\circ}$ XI.42, pour le même style voir $\mathrm{n}^{\circ}$ XI.3, $7, \mathrm{n}^{\circ}$ XI.16, 18, $28, \mathrm{n}^{\circ}$ XI.43 et $\mathrm{n}^{\circ}$ XI.59, pour la chronologie voir p. 3,

121 ; P. A. Mountjoy, Mycenaean Pottery, An Introduction, Oxford, Oxbow Books, 1993, p. 100, fig. 266.

31. Spyropoulos 1969 b, p. 23-24, fig. 6-8 ; Mountjoy 1999 , p. 671 , fig. $255, \mathrm{n}^{\circ} 110$. D'autres chercheurs datent ce skyphos de l'HR III B-C, voir R. Hope Simpson, 
décor des larnakès de cette tombe n'est pas décrit. En revanche, la céramique dans la larnax de la tombe 32 est datée par Th. G. Spyropoulos de l'HR III B ${ }^{32}$. Les inhumations en larnakès doivent par conséquent avoir été pratiquées pendant tout l'HR III B.

La nécropole de Dendron est donc restée près de deux cents ans en fonction; celle de Géphyra, plus de cent ans. D'après le mobilier associé et l'analyse stylistique des larnakès, l'usage de ces contenants funéraires, attesté en premier lieu à Dendron, en dernier lieu à Géphyra, s'étend sur près de cent cinquante ans, de l'HR III A2 à l'HR III B2-C.

\subsection{Cas isolés d'inhumations en larnakès}

Des larnakès en terre cuite sont recensées dans quelques cimetières mycéniens (tableau 1). En Béotie, une larnax de terre cuite en forme de baignoire est signalée à Thèbes, dans la tombe 3 de l'Isménion. Cette tombe est la plus ancienne des six tombes de la période mycénienne dégagées sur cette colline. Elle a sans doute été édifiée à l'HR II B, puis rouverte à l'HR III $\mathrm{B}^{33}$. Le fragment de larnax ne peut toutefois être daté avec certitude. La tombe, rouverte également à l'époque géométrique, contenait deux crânes sur le dernier sol, ainsi que des restes osseux dont des crânes provenant de cinq inhumations et dispersés sur le premier sol.

Dans la grande tombe à chambre de Mégalo Kastelli, située à l'Est de la Cadmée thébaine, les fragments peints d'une larnax en terre cuite ont été dégagés des remblais accumulés à l'intérieur du premier $d_{r o m o s}{ }^{34}$. Des fragments de vases HR III B jonchaient le sol de ce dernier. Une sépulture constituée d'un crâne et d'une pyxide se trouvait dans la partie occidentale de la chambre, la présence d'un squelette est mentionnée au Sud. La tombe à l'architecture imposante est connue pour ses fresques et ses quelques pièces de mobilier découvertes in situ.

Dans le Péloponnèse, les traces d'inhumations en larnakès de terre cuite sont éparses. En Argolide, une larnax en forme de coffre a été découverte dans l'une des trois tombes de la colline de Vraserka près de Prosymna ${ }^{35}$. Ces tombes de l'HR III A-B qui contenaient plusieurs inhumations se situent près de deux autres tombes mycéniennes plus anciennes. De même facture que les larnakès de Tanagra, la larnax de Vraserka est datée, d'après son décor à motifs végétaux et le mobilier céramique de la tombe, de l'HR III A2 ou III B1.

Parmi le grand nombre de tombes mycéniennes fouillées dans la région de Prosymna, la tombe à chambre 17 renfermait une petite larnax en terre cuite peinte qui contenait, sans

O. T. P. K. Dickinson, A Gazetteer of Aegean Civilisation in the Bronze Age, 1 (SIMA, 52), Göteborg, P. Åström, 1979, p. 222-223 Tanagra F63. Nous serions tentées de suivre la datation de P. A. Mountjoy, même si les spirales à tige verticale se rencontrent plus fréquemment à l'HR III B (FM 51). On peut également préciser que la tasse de l'HR III B2-C décrite dans l'ouvrage de P. A. Mountjoy (p. 681, fig. $260, \mathrm{n}^{\circ} 167$ ) provient bien de la tombe $1 \alpha$ de Géphyra, et non de Dendron.
32. Spyropoulos 1971, p. 12.

33. Keramopoullos 1917 , p. 87 , 92, fig. 66 ; S. Symeonoglou, The Topography of Thebes from the Bronze Age to Modern Times, Princeton, Princeton University Press, 1985, p. 237 , site 8 .

34. Spyropoulos 1972 a, p. 309-312.

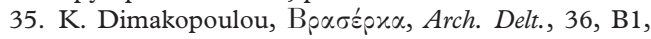
1981 , p. $97-99$, pl. $38 \alpha$; Dimakopoulou 1987, p. 69-78, fig. 1 , pl. 1-10. 


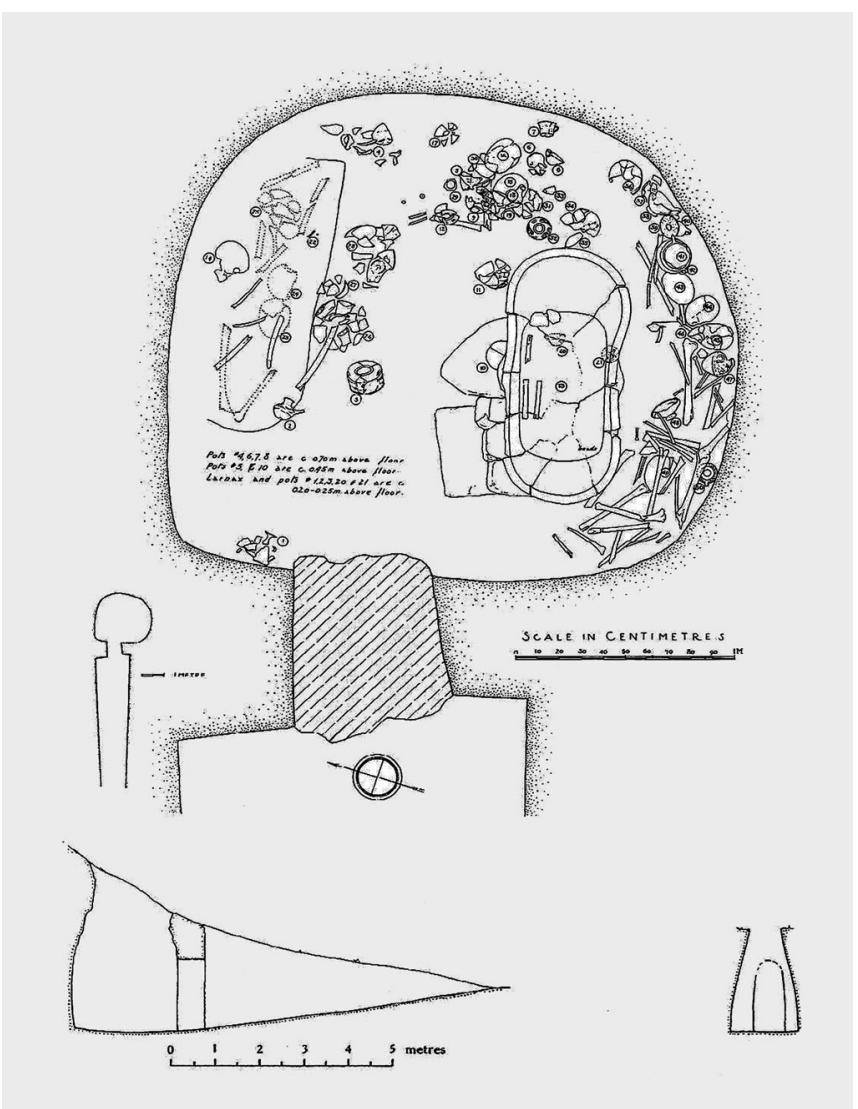

4. Prosymna, tombe XVII.

D'après Blegen 1937, 2, pl. 3

(" Tomb XVIII " mentionnée par erreur sur la planche).

doute, les ossements d'un enfant (fig. 4) ${ }^{36}$. Dans cette tombe, on dénombre treize inhumations qui s'échelonnent sur quatre périodes successives, de l'HR II A à III B1. La larnax en forme de baignoire date probablement de l'HR III. Le décor est en grande partie effacé, mais des traces de peinture noire sont visibles à l'extérieur comme à l'intérieur de la larnax.

À Mycènes, où l'on recense de nombreuses tombes à chambre, la tombe 502, située le long du chemin menant à l'acropole, contenait un grand fragment de terre cuite identifié comme un fragment probable de larnax HR III ${ }^{37}$. Bien que la chambre comprenne au minimum trois inhumations, aucune d'entre elles ne peut être liée à la larnax.

Dans les zones d'habitat, de rares larnakès en forme de baignoire contenaient des inhumations (tableau 2). À Haghios Kosmas, deux larnakès de ce type se trouvaient sous le sol

36. Blegen 1937, p. 53-56, 456, fig. 99, 101, $\mathrm{n}^{\circ} 1206$ L'inhumation en larnax remonte à l'HR III A2, d'après K. S. Shelton, The Late Helladic Pottery from Prosymna
(SIMA, Pocket-Book, 138), Jonsered, P. Åström, 1996, p. 36-41, 193-196, 303, 307.

37. Wace 1932 , p. 3-10, fig. $4, \mathrm{n}^{\circ} 27$ a. 
du porche de la Maison S datée de l'HR III $C^{38}$. Elles renfermaient chacune une inhumation. À Mycènes, près de la Porte des Lions, une larnax contenait peut-être une inhumation dont le contenu composé de quelques ossements et de vases de l'HR III C a été mis au jour hors de la larnax $^{39}$. Finalement, à Ktouri en Thessalie, une larnax en forme de baignoire provient d'une tombe à ciste située à l'Ouest du tertre ${ }^{40}$. Des fragments de vases ont été découverts à l'intérieur, mais aucune inhumation n'est mentionnée. La larnax qui remonte probablement à l'HR III B a été découverte près d'une autre tombe dans une tranchée ayant livré beaucoup d'ossements et de fragments mycéniens. Il est donc possible qu'un groupe de tombes ait avoisiné les maisons.

Tableau 2. - Corpus des inhumations en larnakès découvertes en contexte d'habitat en Grèce continentale*

\begin{tabular}{|c|c|c|c|c|c|c|c|}
\hline & \multicolumn{2}{|c|}{ Tombes en fosse } & \multicolumn{2}{|c|}{ Tombes de type inconnu } & \multicolumn{2}{|c|}{ Autres } & \multirow[t]{2}{*}{ Datation } \\
\hline Sites & $\begin{array}{c}\text { Dénomi- } \\
\text { nation } \\
\text { des tombes }\end{array}$ & $\begin{array}{c}\text { Nombre } \\
\text { de } \\
\text { larnakès } \\
\text { en }\end{array}$ & $\begin{array}{c}\text { Dénomi- } \\
\text { nation } \\
\text { des tombes }\end{array}$ & $\begin{array}{c}\text { Nombre } \\
\text { de } \\
\text { larnakès } \\
\text { en } \\
\text { terre cuite }\end{array}$ & Lieux & $\begin{array}{c}\text { Nombre } \\
\text { de } \\
\text { larnakès } \\
\text { en } \\
\text { terre cuite }\end{array}$ & \\
\hline \multirow[t]{2}{*}{ H. Kosmas } & Tombe 5 & 1 & & & & & HR III C \\
\hline & Tombe 6 & 1 & & & & & HR III C \\
\hline Ktouri & & & $\begin{array}{l}\text { tombe } \\
\text { (dallée) }\end{array}$ & 1 & & & HR III B \\
\hline Mycènes & & & & & $\begin{array}{c}\text { Porte } \\
\text { des Lions }\end{array}$ & 1 & HR III C \\
\hline
\end{tabular}

À Drosia, en Béotie, la larnax découverte dans l'une des tranchées ouvertes en 1989 n’a vraisemblablement pas servi de contenant funéraire ${ }^{41}$. Bien que deux de ces tranchées aient compris des restes de tombes, le fond de la larnax en terre cuite trouvé près de deux murs ne contenait ni ossements ni mobilier liés à la tombe.

Certaines larnakès en forme de baignoire qui ne comprenaient manifestement aucune trace d'inhumation ont été mises au jour en zone d'habitat. Leur fonction semble exclusivement domestique. En Grèce continentale, ces grandes cuves pourvues ou dépourvues de décor sont attestées sur une douzaine de sites, comme à Pylos, Tirynthe ou Mycènes ${ }^{42}$. D'autres

38. Mylonas 1959, p. 52-55, 61-62, dessin 14 .

39. Wace $1921 / 1923$, p. 36, fig. 4 , pl. VI C.

40. Béquignon 1932, p. 148-149, fig. 41-42.

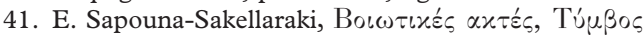

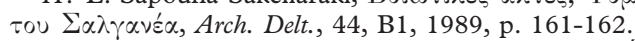

42. Voir P. Darcque, L'habitat mycénien, 1, thèse d'État,
Université de Paris I, 1998, p. 218-221, 328-329 [publiée en 2005] ; Pylos: C. W. Blegen, The Palace of Nestor Excavations of 1958, Part I, AfA, 63, 1959, p. 123-124;

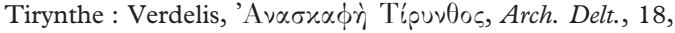
B1, 1963, p. 73, pl. 83-88; Mycènes : French 1961, p. 8889, pl. 14 . 
fragments en terre cuite sont attribués à des larnakès; leur identification demeure toutefois incertaine ${ }^{43}$. En Crète, certaines larnakès ont sans doute servi de baignoires, comme celle du Mégaron de la Reine à Cnossos ${ }^{44}$.

\section{INHUMATIONS EN CONTENANTS DE BOIS}

\subsection{Cas vérifiables par l'analyse de la position des restes osseux}

Une relecture de la documentation des cimetières de l'Agora d'Athènes, d'Éleusis et de Pérati met en évidence la présence de contenants funéraires en bois (tableau 1). Le cimetière d'Éleusis, en usage de l'HM à la période classique, compte un grand nombre de tombes rectangulaires construites, quelques tombes à chambre et des tombes à ciste ${ }^{45}$. De même, le cimetière de l'Agora d'Athènes qui couvre la période de l'HM au début de la période archaïque se compose de tombes à chambre, de tombes à ciste et de tombes en fosse ${ }^{46}$. Enfin, le cimetière de Pérati comprend cent quatre-vingt-douze tombes à chambre et vingt-six tombes en fosse, datées de l'HR III B à l'HR III C1 ${ }^{47}$.

La présence de contenants en matière périssable peut être déterminée par l'observation de la position des ossements ${ }^{48}$. Les informations à ce sujet doivent être récoltées sur le terrain. En ce qui concerne les anciennes publications, il faut se reporter à des documents photographiques susceptibles de montrer la position des restes osseux. Cette observation permet de déterminer la position originelle des défunts. Ainsi les contenants périssables sont mis en évidence par différents effets de délimitation linéaire, de butée ${ }^{49}$ et de constriction ${ }^{50}$. Ces trois critères d'identification sont confortés par d'autres, plus généraux, qui plaident en faveur d'une décomposition en espace vide, tels que l'absence de connexions anatomiques.

Nous pouvons ainsi mettre en évidence que certains individus ont été déposés à l'intérieur soit d'un cercueil, à Éleusis et à l'Agora d'Athènes, soit d'une larnax en bois dans les cimetières d'Éleusis et de Pérati. En effet, les squelettes qui se présentaient avec les genoux en extension durent être introduits à l'intérieur d'un cercueil de forme rectangulaire, comme l'enfant (3) de la tombe $\Lambda \pi 4$ d'Éleusis (fig. 5) ${ }^{51}$. Toutefois, l'individu de la tombe $H \pi 5$

43. Vermeule et Karageorghis 1982, p. 209, 221, $\mathrm{n}^{\circ}$ VII. 3 (Mycènes), VII K (Thèbes), XI. 30 et XI. 31 (Tirynthe).

44. A. Evans, The Palace of Minos, III, Londres, MacMillan, 1930, p. 385, fig. 256.

45. Mylonas 1975 , vol. B, p. 301.

46. Immerwahr 1971 , p. 98 et 103 ; S. A. Immerwahr, Early Burials from the Agora Cemetery, Princeton, The American School of Classical Studies at Athens, 1973, p. 1.

47. S. E. Iakovidis, Excavations of the Necropolis at Perati, Los Angeles, Institute of Archaeology, Univ. of California, 1980, p. 4-5, 108

48. La méthode a été définie dans Duday 1995. Actuellement, cette méthode porte le nom d'archéothanatologie.
49. Concerne, dans une sépulture, les os qui s'alignent et sont maintenus en équilibre instable en prenant appui contre une structure qui empêche leur chute.

50. Notamment la mise à la verticale des clavicules et le non-basculement en arrière des os coxaux.

51. Nous avons observé un effet de constriction dans l'attitude générale du squelette. Celui-ci est mis en évidence par l'obliquité des côtes et la rotation de l'humérus droit en face latérale, dues à la projection en avant des épaules et liées à l'exiguïté d'un contenant sans doute périssable. De plus, nous avons un effet de délimitation linéaire au niveau des faces latérales de ce sujet et du mobilier à gauche. 


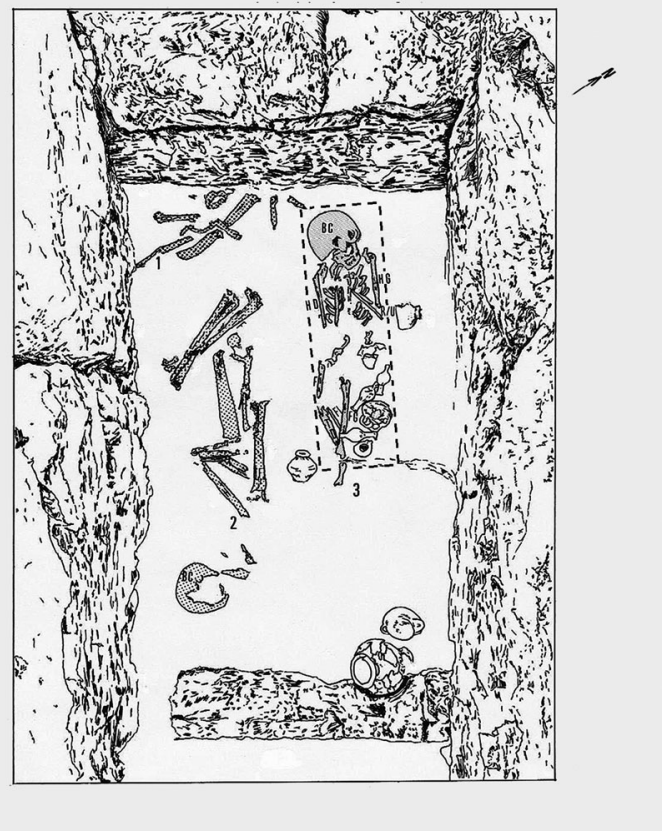

5. Cimetière d'Éleusis, sépulture $\Lambda \pi 4$.

Dessin de S. Farrugio. D'après Mylonas 1975, Г, pl. 153.

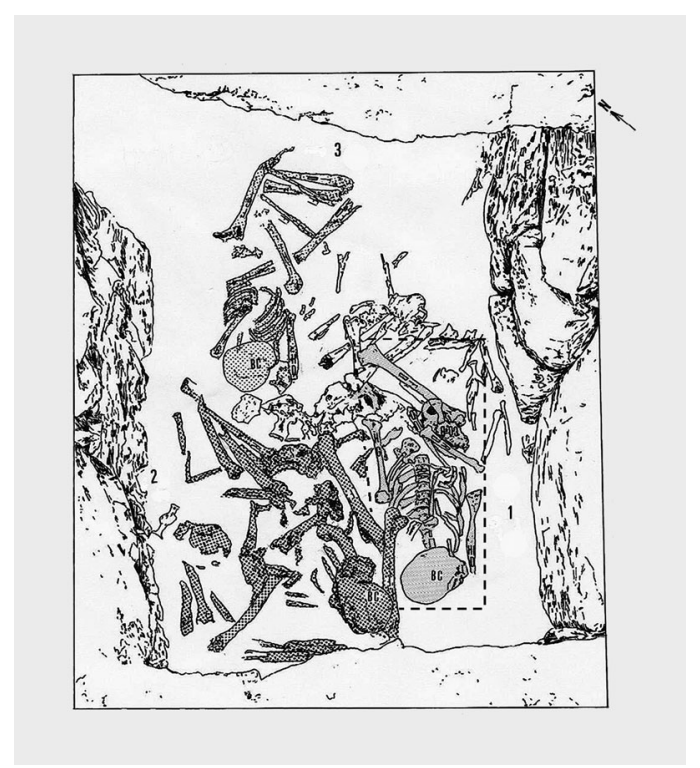

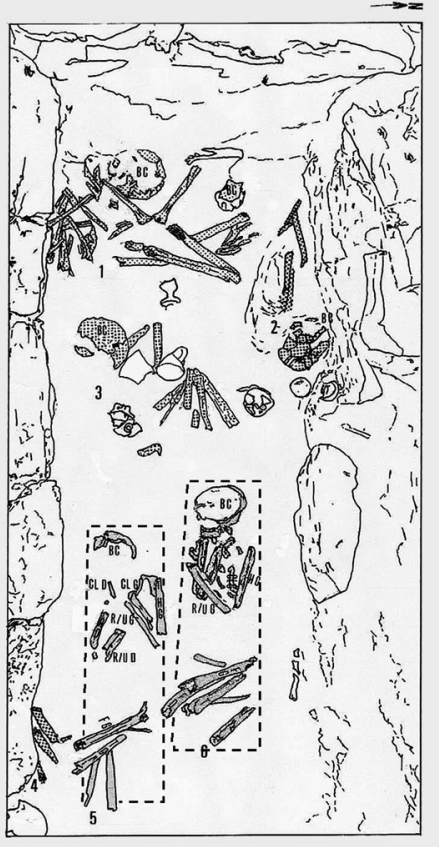

6. Cimetière d'Éleusis, sépulture $\mathrm{E} \pi 1$. Dessin de S. Farrugio.

D'après Mylonas 1975, Г, pl. 35.

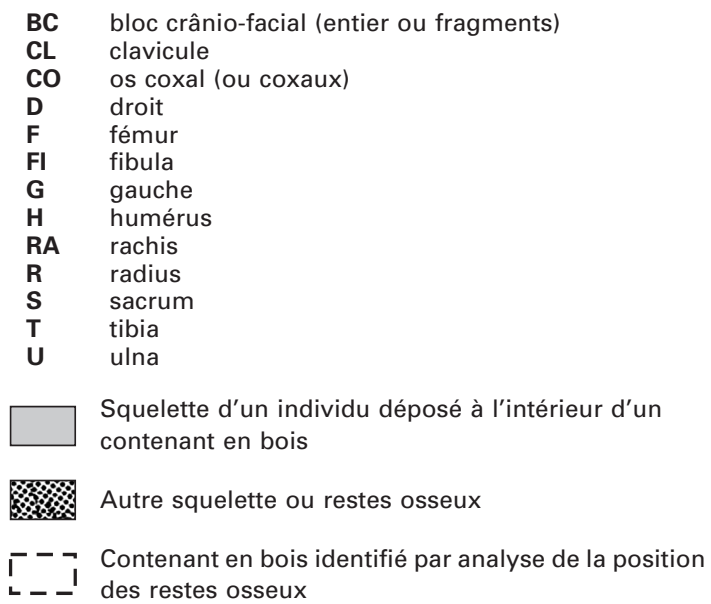

7. Cimetière d'Éleusis, sépulture $\Theta \pi 5$.

Dessin de S. Farrugio. D'après Mylonas 1975, , pl. 106. 
d'Éleusis se trouvait sans doute à l'intérieur d'un cercueil de forme trapézoïdale, comme en témoigne la position des os du bassin ${ }^{52}$.

La tombe à chambre XL de l'Agora contenait deux individus (C et $\mathrm{D})$ qui devaient être chacun introduits à l'intérieur d'un cercueil en bois, probablement de forme rectangulaire, comme l'attestent les traces de bois que signale S. A. Immerwahr. Des effets de délimitation linéaire des parties latérales (gauche pour C, gauche et droite pour D) indiquent ce type de contenant ${ }^{53}$.

En revanche, il est fort probable que certains sujets furent introduits à l'intérieur d'un contenant fait de planches de bois reproduisant la forme et les dimensions, légèrement supérieures à $1 \mathrm{~m}$, des larnakès en terre cuite. Ces individus devaient avoir les genoux fléchis audessus du fond d'un contenant périssable. Un des deux membres inférieurs prenait alors appui contre l'un des grands côtés du contenant, tandis que le genou du second membre inférieur reposait contre la cuisse ou la jambe du premier membre.

Ce cas est illustré dans la tombe $57 \mathrm{du}$ cimetière de Pérati, et, dans les tombes $\mathrm{E} \pi 1$ (fig. 6), $\Theta \pi 4, \Theta \pi 5$ (fig. 7), $M \pi 6$ et $M \pi 9$ du cimetière d'Éleusis, nous constatons que certains des défunts furent introduits à l'intérieur de larnakès en bois. De plus, dans ce dernier cimetière, certains sujets, dont nous avons observé une mise à la verticale des clavicules, purent être introduits à l'intérieur d'un linceul étroit (collé ou cousu), comme les individus 5 et 6 de la tombe $\mathrm{E} \pi 1$ (fig. 6) et celui de la tombe M $\pi 9$ (situé le long de la paroi Sud ${ }^{54}$ ). Cependant, il est fort probable que l'individu de la dernière tombe ait été déposé dans un contenant assez large et que la contrainte rendue visible par la mise à la verticale des clavicules soit due à une chemise très ajustée.

En ce qui concerne la tombe 8 de Dendra, datée par A. W. Persson de l'HR II, les fouilleurs ont identifié d'après les traces de bois décomposé un cercueil mesurant $1,90 \mathrm{~m}$ de long sur $0,50 \mathrm{~m}$ de large $\mathrm{e}^{55}$. La couche gris-bleu qui couvre le tout est souvent considérée comme des restes de peinture jugée comparable à la peinture bleue des cercueils découverts en Crète ${ }^{56}$. Le squelette fut mis au jour avec les restes de bois. Cependant, l'évaluation de cette hypothèse sur la base des documents photographiques demeure impossible, étant donné l'état de la documentation. Des armes et un casque ont aussi été découverts dans la tombe. Attribuer l'ensemble du mobilier à l'inhumation en contenant est discutable, puisque la tombe contenait probablement quatre inhumations ${ }^{57}$.

52. Cet individu fut vraisemblablement introduit dans un cercueil de bois étroit de forme trapézoïdale. En effet, la fermeture ou la faible ouverture du bassin au niveau des symphyses pubiennes suggère la forme trapézoïdale d'un cercueil plus large au niveau des épaules du squelette et plus étroit au niveau des pieds.
53. Immerwahr 1971, p. 101, pl. 59, 90.

54. Mylonas 1975, vol. B, p. 199.

55. Persson 1942, p. 40, 111, fig. 44.

56. Voir également Hägg et Sieurin 1982, p. 180.

57. Persson 1942, p. 41, 43-45. 


\subsection{Cas invérifiables par un nouvel examen de la documentation}

Il est impossible de réexaminer les tombes du Cercle A de Mycènes et celles du cimetière de Prosymna à partir des documents disponibles. Aussi proposons-nous, en l'absence de photographies détaillées des ossements, une simple étude critique des publications.

À Mycènes, les tombes I, III, IV et V ont livré des restes de bois. Pour la tombe III, ces restes sont interprétés par R. Hägg et F. Sieurin, ainsi que par P. Muhly, comme étant ceux d'un cercueil. Aucune distinction entre cercueil, brancard ou tout autre objet n'a pu être effectuée pour les trois autres tombes ${ }^{58}$. Cependant, plusieurs chercheurs, en se fondant sur l'emplacement du mobilier, démentent tout usage de cercueilis9.

À Prosymna, les tombes X, XXIX et XLII se situent au lieu-dit Yérogalaro, tandis que la tombe XVII se trouve plus à l'Ouest, près de l'Héraion. Les restes de bois recueillis dans les tombes X, XXIX et XLII appartiendraient, selon C. W. Blegen, à des brancards sur lesquels les défunts étaient allongés. Précisons que les traces de bois dans la tombe XXIX correspondaient, d'après l'auteur, à un brancard mesurant environ $1,20 \mathrm{~m}$ de long sur $0,70 \mathrm{~m}$ de large ${ }^{60}$. Comme nous l'avons vu précédemment, la tombe XVII contenait une larnax en terre cuite.

Le cas des cimetières de Tanagra est problématique. Aucune larnax en bois n'est signalée dans les tombes, mais, d'après Th. G. Spyropoulos, il est possible que des larnakès en bois aient contenu des inhumations à Dendron ${ }^{61}$. L'état de la documentation ne permet pas de se prononcer à ce sujet.

À Thèbes, la tombe 24, située au Sud-Ouest de Kolonaki, contenait peut-être une larnax en bois. Toutefois, il n'en reste aucune trace, si ce n'est en négatif, sur des fragments de mortier. Selon le fouilleur, elle était peut-être destinée à un enfant ${ }^{62}$. Toutefois, deux inhumations ont été découvertes dans la tombe, ce qui rend cette attribution incertaine.

$\mathrm{Au}$ total, on recense des inhumations en contenants funéraires dans onze cimetières de Grèce continentale (voir carte). Une forte concentration se rencontre en Béotie et, dans une moindre mesure, en Attique. En Béotie, des larnakès en terre cuite ont été trouvées in situ, mais l'état de la documentation se prête peu à un nouvel examen de leur contenu. En revanche, en Attique, l'usage de larnakès et de cercueils en bois est principalement confirmé par l'étude de la position des restes osseux, alors que les contenants en matière périssable n'ont laissé que très peu de traces. On ne relève dans aucun cimetière mycénien l'usage simultané de contenants en terre cuite et en bois.

Les contenants funéraires ont été découverts dans des catégories de tombes distinctes. Par exemple, à Dendron et à Géphyra, près de Tanagra, trente-huit tombes à chambre renfermaient des larnakès en terre cuite. À Éleusis, il est possible d'identifier des inhumations en contenants dans sept tombes rectangulaires construites. Dans l'état actuel des recherches,

58. Hägg et Sieurin 1982, p. 179 ; Muhly 1996, p. $206-208$.

59. Long 1974 , p. 17 ; voir la discussion dans Persson 1942, p. 113-114.
60. Blegen 1937, p. 76,150 et 199

61. Spyropoulos 1974, p. 20-21 ; Th. G. Spyropoulos,

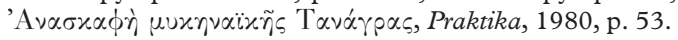
62. Keramopoullos 1917, p. 184-187. 
la présence d'inhumations en contenants dans d'autres cimetières de Grèce continentale demeure sporadique. Quant au Dodécanèse, aux Cyclades et à Chypre, des larnakès en pierre ou en terre cuite ont été découvertes dans des tombes à chambre et à puits, mais leur usage reste également peu attesté ${ }^{63}$.

\section{REMARQUES SUR LES PRATIQUES ET RITES FUNÉRAIRES LIÉS AUX INHUMATIONS EN CONTENANTS}

\subsection{Mise en larnax et soins funéraires}

Pour la première fois en Grèce continentale, des représentations liées au domaine funéraire sont peintes sur les larnakès de Tanagra. Il s'agit de figures féminines portant les mains à la tête et de personnages couchés dans un contenant ou sur un support. Ces scènes apparaissent sur les parois des larnakès. Des représentations similaires se rencontrent sur les vases à la période géométrique; leur lecture est principalement influencée par les textes homériques ${ }^{64}$. Quatre siècles séparent toutefois l'effondrement de la civilisation mycénienne de l'époque géométrique, et la signification des scènes peut varier à travers les époques ${ }^{65}$. Sur le continent, on n'a, jusqu'à présent, pas décelé d'inhumations en contenants à l'époque géométrique, période où les incinérations se répandent.

D'après les représentations de défunts figurant sur les larnakès, il est difficile de déterminer l'âge et le sexe des individus. À Tanagra, le fouilleur rapporte la présence d'adultes et d'enfants inhumés en larnakès. Dans la tombe I $\alpha$ de Géphyra, un sujet adulte était déposé sur le dos en position contractée dans une larnax et un skyphos fut retrouvé contre la partie antérieure du thorax ${ }^{66}$. Le seul objet personnel associé à un défunt dans une larnax n'a pas été découvert lors des fouilles officielles ${ }^{67}$.

L'inhumation des défunts avec leurs armes ou bijoux était pourtant connue dans le monde égéen. Dans une larnax de Pangalochori en Crète, une femme enceinte portait des objets de parure ${ }^{68}$. Selon une nouvelle observation de la documentation, la défunte fut déposée

63. Larnakès de terre cuite: Rhodes, Ialysos, T. 81 (à puits): G. Jacopi, Nuovi scavi nella Necropoli Micenea di Jalisso, Ann. Sc. Atene, 13-14, 1930-1931, p. 311-312, fig. 59 ; Karpathos, t. à chambre : O. Zacharidou, $\Theta \alpha \lambda \alpha \mu_{0-}$

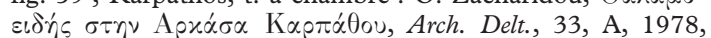
p. 274, fig. 10, pl. $83 \alpha-\beta$; Naxos, Grottes : Vermeule 1965, p. 124 , n. 3 ; Larnakès en pierre: Chypre: H. W. Catling, V. Karageorghis, Minoika in Cyprus, ABSA, 55, 1960, p. 127 ; Égine, Kolonna : G. Welter, Aeginetica XIII-XXIV, $A A, 1938$, p. 511-512; Kephallenia, Kontogenada : S. Ma-

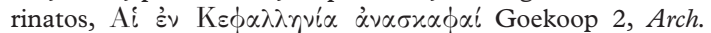
Eph., 1933, p. 70, 79, fig. 22 ; Kephallenia, Parisata Palis :

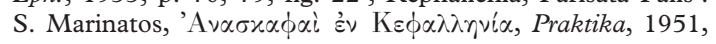
p. 186.

64. Voir Spyropoulos 1974, p. 21-22 ; C. Sourvinou Inwood, Minoan and Mycenaean Afterlife Beliefs and their Rele- vance to the Homeric Underworld, Diss. Phil. Oxford, St. Hugh's College, 1973, p. 525. Pour les funérailles chez Homère, voir Iliade, XXIII, et Odyssée, IV, v. 563-568.

65. Pour une lecture critique des représentations funéraires, voir Immerwahr 1995, p. 109-121 ; Cavanagh et Mee 1995, p. 45-61 ; Benzi 1999, p. 215-233.

66. Spyropoulos 1969 b, p. 21, 23, fig. 3 .

67 . Vermeule 1965 , p. 125 . L'A. relève qu'un des squelettes découvert dans une larnax lors de fouilles illicites portait un collier de perles en stéatite dont la pierre centrale était gravée. Cette pierre correspond peut-être au sceau n ${ }^{\circ} 682$ décrit dans le CMS V .2, p. 557, conservé au musée de Thèbes, inv. 741 (?).

68. K. Baxevani-Kouzioni, S. Markoulaki, Une tombe à chambre MR III à Pankalochori, $B C H, 120$, 1996, p. 641-647, 660-665, fig. 31-34. 

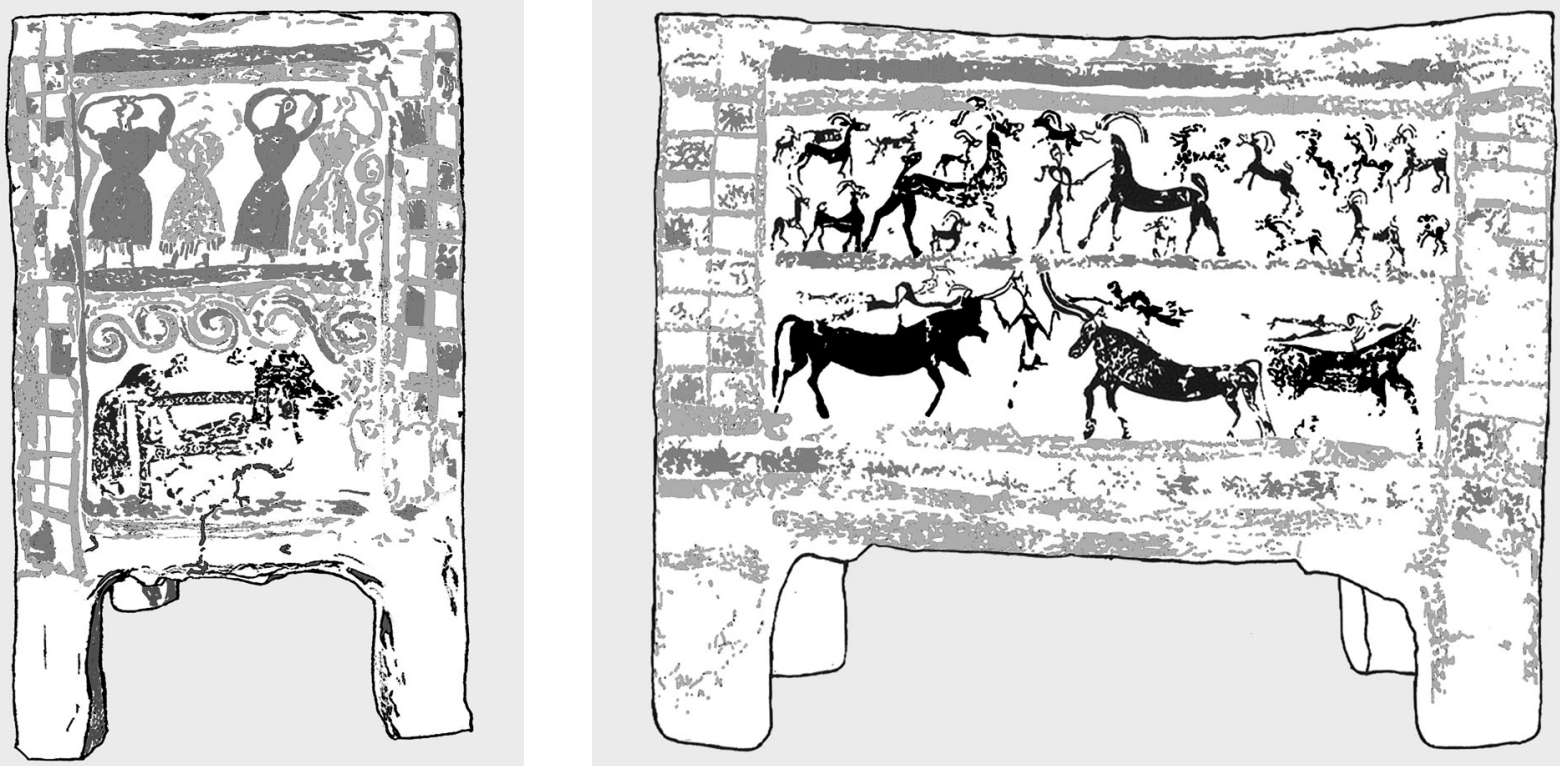

8 a. Géphyra, tombe 22 : scène figurée du petit côté de la larnax. D'après Spyropoulos 1970 b, p. 195, fig. 15.

8 b. Géphyra, tombe 22 : scène figurée du grand côté de la larnax. D'après Spyropoulos 1970 b, p. 196, fig. 16.

de trois quarts antérieur avec une flexion des genoux dans la larnax. Un linceul tenu par des aiguilles en os enveloppait peut-être son corps.

Sur une scène figurée de la larnax trouvée dans la tombe 3 de Dendron, deux personnages féminins semblent couvrir d'un linceul le corps du défunt reposant sur une larnax ou un support; la scène dépeinte tend donc à confirmer l'emploi d'un linceul (fig. 2) ${ }^{69}$. D'autres représentations comportent une larnax, mais on ne peut identifier avec certitude un linceul. Sur la larnax de la tombe 22 de Géphyra, deux femmes touchent le défunt couché dans une larnax, ce dernier était peut-être enveloppé d'un linceul (fig. $8 \mathrm{a}$ ) $^{70}$.

Il est probable que d'autres pratiques précèdent la mise en larnax. Cependant, c'est en Crète que les témoignages se montrent les plus parlants. Le défunt et les soins funéraires qui lui sont rendus sont figurés sur le couvercle d'une larnax de Pigi Rethymnou datée du MR III A2-B (fig. 9) ${ }^{71}$. Un corps est étendu sur une table. Sur la gauche de la scène, une figure en robe s'approche de la table, tandis qu'à droite une figure en tunique courte tend les bras vers la tête du défunt.

69. Spyropoulos 1970 a, p. 34-35, pl. $48 \alpha$.

70. Spyropoulos $1969 a$, p. 14 , pl. $13 \alpha, 14 \beta$; Spyropoulos 1970 b, p. 190-197, fig. 15-17.
71. G. Tzedakis, B. Nouòs Pżúurne, Arch. Delt., 24, B2, 1969, p. 435-436, pl. 443; Baxevani 1995, p. $17-18,21$, fig. $1-14$. 
Dans le cas des individus déposés à l'intérieur de larnakès en terre cuite ou en bois, nous supposons que le fléchissement des genoux imposait que le dépôt du cadavre s'opère soit immédiatement après la mort, soit quelques jours plus tard ${ }^{72}$. En effet, comme le firent remarquer M. Orfila et L.-V. Thomas, la rigidité cadavérique survient en moyenne trois ou quatre heures après le décès et se généralise en douze heures environ. Ce processus commence par le visage. Par la suite, il atteint la nuque, puis le tronc et les membres. Au moment où la putréfaction débute, la rigidité cadavérique disparaît dans l'ordre inverse de son établissement. Ce changement s'opère entre vingt-quatre et soixante-douze heures après la mort de l'individu. Notons que, si la rigidité cadavérique est interrompue après la treizième heure, elle ne se reproduit plus. Cette contrainte influait sur le temps qui devait s'écouler entre la mort de la personne et son inhumation: le défunt devait être introduit dans la larnax avant que n'intervienne la rigidité cadavérique ou après sa disparition.

\subsection{Pratiques cérémonielles}

Il est dommage que les fragments de larnax peints provenant du dromos de la tombe de Mégalo Kastelli ne soient pas décrits. Cette grande tombe de Thèbes était décorée de peintures murales ${ }^{73}$. Les parois du stomion comportaient une représentation figurée et deux figures féminines les bras levés ornaient le côté droit de l'entrée. Un brancard était peut-être peint entre ces figures qui, comme les personnages féminins sur les larnakès de Tanagra, adoptent une attitude de deuil. Cependant, sur le plan stylistique, les figures de la tombe de Mégalo Kastelli ne leur ressemblent guère, mais rappellent davantage les porteuses d'offrandes de la fresque de la procession de la Maison de Kadmos à Thèbes ${ }^{74}$.

Sur les larnakès de Tanagra, un grand nombre de figures portent les mains à la tête, la bouche ouverte dans une attitude typique de deuil. Elles accompagnent à deux reprises les scènes illustrant le défunt déposé dans la larnax, mais apparaissent également seules. Peintes le plus souvent la bouche ouverte et le cou parfois griffé, elles devaient exprimer leur peine par des lamentations funèbres ${ }^{75}$. Les figures féminines en deuil traduisent une attitude conventionnelle face à la mort, adoptée dans l'art funéraire mycénien sans doute dès l'HR III A2 ${ }^{76}$. Leur arrangement en frise souligne l'aspect cérémoniel de la scène. Sur les larnakès, la composition illustre peut-être un rite ou un moment particulier lors d'une cérémonie funéraire (fig. 3$)^{77}$.

72. M. Orfila, Traité de médecine légale, 1, Paris, Labé, 1848 , p. 483 ; L. V. Thomas, Le cadavre, De la biologie à l'anthropologie, Bruxelles, Éd. Complexe, 1980, p. 21.

73. Spyropoulos 1972 a, p. 310-312, pl. 254-255; Kontorli-Papadopoulou 1996, n 109, pl. 130.

74. Dimakopoulou et Konsola 1981, p. 50, pl. 21 ; Kontorli-Papadopoulou 1996, n 106, pl. 129.

75. Il s'agit de larnakès provenant aussi bien de Dendron que de Géphyra, voir Cavanagh et Mee 1995, p. 48-49, fig. 1, 10.
76. Voir Vermeule 1965, p. 142. Déterminer s'il s'agit d'une expression naturelle de peine ou d'une codification des gestes reste problématique selon la composition des représentations dans lesquelles s'inscrivent les figures en deuil.

77. Pour les larnakès de la tombe 6 à Géphyra et de la tombe 16 à Dendron, voir Spyropoulos 1970 b, p. 193, fig. 9-10, 12 ; Spyropoulos 1973, p. 21 , pl. $11 \beta$. 
L'implication des hommes dans des cérémonies funéraires reste moins attestée. Sur les larnakès de Géphyra, les personnages masculins portent des tuniques, parfois des casques, certains semblent barbus, d'autres ont la bouche ouverte ${ }^{78}$. Ils ne paraissent ni être armés ni porter de cuirasse à l'image de l'armement trouvé dans la tombe 12 de Dendra de l'HR III A1 ${ }^{79}$. Les hommes n'accompagnent pas les défunts peints sur les larnakès de Tanagra. Seul un vase mycénien provenant d'Élide montre ce type d'association : un personnage masculin est représenté près d'un défunt. D'après $\mathrm{O}$. Vikatou, ce dernier est couché sur un lit funèbre, mais il pourrait s'agir d'un contenant caractérisé par un trait arqué passant au-dessus du défunt (fig. 10) ${ }^{80}$.

Par ailleurs, on peut s'interroger sur les liens entre représentations de chasse et domaine funéraire. Un homme armé d'une épée menace une chèvre sur la larnax de la tombe 22 de Géphyra (fig. $8 b$, registre supérieur) ${ }^{81}$. Les seuls documents de comparaison, ornés de scènes de chasse, proviennent de Crète, comme la larnax d'Épiskopi Iérapétras et celle de Maroulas Rethymnis $^{82}$. Les animaux sont fréquemment représentés sur les larnakès d'Arménoi, parfois face aux chasseurs comme sur la larnax de la tombe $11^{83}$. Il se peut que les animaux chassés aient constitué le repas funèbre en Grèce continentale comme en Crète.

Il est intéressant de noter que des restes osseux de chèvres, incluant des crânes, sont signalés dans des tombes à Tanagra. On en a découverts dans les remblais de la tombe 1 de Géphyra qui contenait également des morceaux de larnakès et de la céramique de l'HR III B. Th. G. Spyropoulous rappelle qu'en Grèce la découverte d'ossements animaux dans des tombes n'est pas exceptionnelle ${ }^{84}$. On en relève notamment la présence dans plusieurs tombes à Pérati ${ }^{85}$. Bien qu'aucune scène d'offrande ni de sacrifice ne soit représentée sur les larnakès de Grèce continentale, on ne peut exclure que des sacrifices aient eu lieu lors de funérailles, comme l'indiquent les ossements dans des tombes de Crète ${ }^{86}$.

En Béotie, d'autres témoignages archéologiques viennent confirmer l'existence d'un repas funèbre lors de l'inhumation d'un défunt. À Dendron, dans la tombe 76, un vase contenait des matières brûlées ${ }^{87}$. Un cas similaire est attesté dans l'imposante tombe à chambre de Mégalo Kastelli, où une grande amphore renfermait des cendres et les ossements d'un petit animal ${ }^{88}$. De plus, des fragments de coupes et d'un réchaud datés de l'HR III B jonchaient le sol de son dromos, où ont été mis au jour les fragments de larnax.

78. Pour les tombes 6 et 60 de Géphyra, voir Spyropoulos $1969 a$, p. 7-12, pl. 4 , $6 \alpha$; Spyropoulos 1971, p. 13, pl. $17 \alpha-\beta, 18 \alpha$; Dimakopoulou et Konsola 1981, p. 83.

79. N. M. Verdelis, Neue Funde von Dendra, $A M, 82$, 1967 , p. 5, fig. 2 ; P. Âström, Das Panzergrab von Dendra, Bauweise und Keramik, $A M$, 82, 1967, p. 54-66.

80. Vikatou 2001, p. 273-284.

81. Spyropoulos 1970 b, p. 190-197, fig. 16. L. V. Watrous suggère que le défunt retrouvait dans l'au-delà des activités semblables à sa vie terrestre, voir L. V. Watrous, The Origin and Iconography of the Late Minoan Painted Larnax, Hesperia, 60, 1991, p. 301. D'après M. Benzi, il se peut que la scène représente un rite de passage, voir Benzi 1999 , p. 226.

82. Kanta 1980 , pl. $63, \mathrm{n}^{\circ} 1-5$, et pl. $102, \mathrm{n}^{\circ} 3-4$.

83. Tzedakis 1971 , p. 217 , fig. 4 , pl. III3.
84. Spyropoulos 1969 b, p. 22 ; Spyropoulos 1969 a, p. 14-15; Spyropoulos 1970 b, p. 191. Des os de chèvres proviennent de la Tombe Iota du Cercle $\mathrm{B}$ de Mycènes.

85. Y. Hamilakis, Eating the Dead: Mortuary Feasting and the Politics of Memory in the Aegean Bronze Age Societies, dans K. Branigan éd., Cemetery and Society in the Aegean Bronze Age, Sheffield, Sheffield Academic Press, 1998, p. 119-120.

86. Immerwahr 1995, p. 116 ; Y. Hamilakis, A Footnote on the Archaeology of Power, Animal Bones from a Mycenaean Chamber Tomb at Galatas, NE Peloponnese, $A B S A, 91,1996$, p. 153-166.

87. Spyropoulos 1974 , p. 9.

88. Spyropoulos 1972 a, p. 309-312 ; W. Cavanagh, C. Mee, A Private Place, Death in Prehistoric Greece, SIMA, 125, Jonsered, P. Åström, 1998, p. 68, 74, n. 179. 
9. Pigi Rethymnou, tombe : scène figurée sur le couvercle d'une larnax.

D'après Baxevani 1995, p. 18 , fig. 10

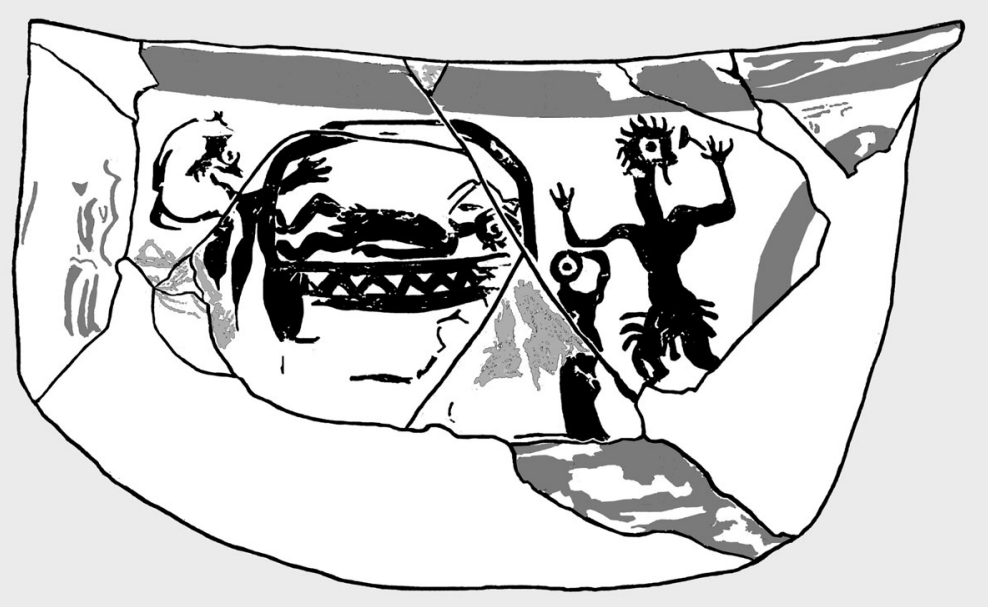

10. Haghia Triada en Élide, tombe 5 : scène figurée d'un cratère.

D'après Vikatou 2001, pl. 27.2 .
11. Dendron, tombe 36 : scène figurée de la larnax. D'après Spyropoulos 1973, pl. $10 \beta$.

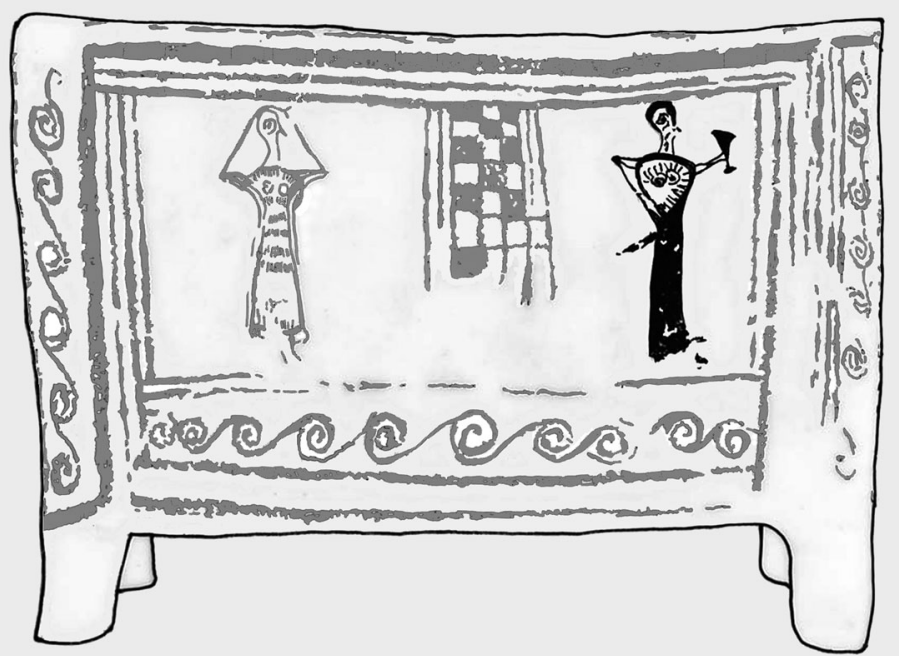


Accompagnant le repas ou destinée à des libations, la boisson jouait sans doute un rôle significatif lors d'une inhumation, que le défunt soit déposé sur le sol de la chambre ou introduit dans une larnax ${ }^{89}$. De nombreux fragments de coupes ont été découverts dans les tombes en Grèce continentale ${ }^{90}$. Des fragments ont été dégagés dans la tombe 3 de l'Ismenion et à l'entrée de la tombe 24 de Kolonaki, près de Thèbes ${ }^{91}$. La première contenait une larnax en terre cuite, la deuxième peut-être un contenant en bois. Des fragments de coupes proviennent aussi des tombes 77, 78 et 94 de Dendron de l'HR III B, qui ne contenaient toutefois pas de larnakès $^{92}$. En Grèce continentale et en Crète, des représentations peintes sur les larnakès et les vases font écho aux découvertes archéologiques, comme les personnages tenant une coupe sur la larnax de la tombe 36 à Dendron (fig. 11), sur la larnax d’Épiskopi Iérapétras, en Crète, et sur le cratère d'Haghia Triada, en Élide (fig. 10) ${ }^{93}$.

\subsection{Cadre des funérailles et domaine de la croyance}

Si des cérémonies funéraires existaient à l'époque mycénienne, déterminer le cadre de leur déroulement s'avère problématique. La représentation de femmes aux fenêtres est un thème iconographique bien connu dans le monde égéen. Elle s'inscrit dans le domaine funéraire en décorant une larnax de la tombe 6 de Géphyra, alors qu'elle est représentée en contexte palatial à Mycènes ${ }^{94}$. Par ailleurs, on compte un autre motif architectural peint sur une des larnakès de la tombe 51 de Géphyra. Il s'agit du pilier qui, emprunté au répertoire iconographique crétois, est considéré par les chercheurs comme la représentation d'un objet de culte ${ }^{95}$. Il pourrait toutefois, en tant qu'élément architectural, indiquer la présence d'un sanctuaire ou d'une tombe.

Découverts près des nécropoles de Tanagra, deux habitats de l’Âge du Bronze présentent des traces d'occupation dès l'HR III A et pendant tout l'HR III $\mathrm{B}^{96}$. Cependant, les rapports de fouilles ne fournissent que peu d'informations concernant leurs structures. On ne peut identifier les lieux où se déroulaient des activités relatives à la préparation du corps ou à la déposition en tombe.

Si des jeux funèbres incluaient des acrobaties au-dessus d'un taureau (taurokathapsie) et des courses de chars qui avaient lieu lors des funérailles, ils avaient peu de chance de se dérouler dans les nécropoles. Ces jeux n'ont laissé aucune trace in situ. Seule la scène peinte sur la

89. Pour l'interprétation de cette pratique rituelle, voir M. Andronikos, Totenkult, Archaeologia Homerica, III, W, Göttingen, Vandenhoeck \& Ruprecht, 1968, p. 93 ; N. Marinatos, Minoan Sacrificial Ritual, Cult Practice and Symbolism, Skrifter, IX, Stockholm, P. Åström, 1986, p. 26-27.

90. W. Cavanagh, Innovation, Conservatism and Variation in Mycenaean Funerary Ritual, dans K. Branigan éd., Cemetery and Society in the Aegean Bronze Age, Sheffield, Sheffield Academic Press, 1998, p. 106-107.

91. Keramopoullos 1917, p. 93, 184.

92. Spyropoulos 1974, p. 10 ; Spyropoulos 1975, p. 422.
93. Spyropoulos 1973 , p. 21 , pl. $10 \beta$; Kanta 1980, pl. $63, \mathrm{n}^{\circ} 1-5$; Vikatou 2001, p. 275 , fig. 1.

94. Spyropoulos 1969 a, p. 7-12, pl. $6 \beta$; pour les peintures murales de Mycènes, voir KontorliPapadopoulou 1996, n 66, pl. 88.

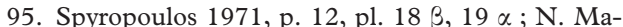
rinatos, Minoan Religion, Ritual, Image, and Symbol, Columbia, University of South Carolina Press, 1993, p. 87.

96. Spyropoulos 1973, p. 17, 19 ; Spyropoulos 1974 , p. 14-17, fig. 8 ; Ergon, 1974, p. 13-14 ; Spyropoulos 1975 , p. 420 , fig. 4 ; Ergon, 1975, p. 18-20. 
larnax de la tombe 22 de Géphyra (fig. $8 b$, registre inférieur) suggère l'existence de ce type de manifestations que l'on ne peut donc proposer qu'à titre d'hypothèse ${ }^{97}$.

Les taurokathapsies et les scènes de char sont également interprétées comme étant des activités du défunt, aussi bien de son vivant que dans l'au-delà, parfois comme étant une prouesse effectuée lors de jeux à caractère initiatique ${ }^{98}$. Cependant, les taureaux sur la larnax de la tombe 16 de Dendron ne sont pas représentés dans une scène de taurokathapsie ${ }^{99}$. De plus, les fragments de larnakès décorés de chars sont découverts en contexte d'habitat à Mycènes et peut-être à Tirynthe ${ }^{100}$. C'est seulement en Crète que des chars sont peints sur des larnakès provenant de tombes de Zaphèr Papoura, d'Épiskopi Iérapétras et de Kavrochori. Les chars s'inscrivent toutefois davantage dans une scène de chasse, tandis que, sur la larnax d'Haghia Triada, ils sont conduits par des personnages féminins, certainement des déesses ${ }^{101}$.

Tenter d'interpréter l'environnement des scènes figurées sur les larnakès de Tanagra demeure difficile. De nombreuses représentations se rapportent davantage au domaine de la croyance. Sur la larnax de la tombe 51 de Géphyra, un sphinx touche le pilier ; sur deux larnakès de Tanagra, des oiseaux géants apparaissent auprès de figures humaines ${ }^{102}$. Toutefois, il est impossible d'attribuer avec certitude une nature divine ou profane aux personnages. Sur les larnakès de Tanagra, les figures aux bras levés représentent, suivant les interprétations, une divinité en épiphanie, un esprit venu chercher l'âme du défunt ou l'âme même du défunt ${ }^{103}$. En Crète, cette question concerne également certaines larnakès de Cnossos et d'Arménoi ${ }^{104}$.

Les oiseaux et les animaux ailés dans le monde crétois sont souvent liés à une divinité. En Grèce continentale, leur connotation funéraire est soulignée. Des oiseaux sont peints sur deux larnakès de Tanagra ${ }^{105}$. Dans les tombes 6 et 60 de Géphyra, des éléments en terre cuite, représentant des êtres ailés fixés ou modelés sur des disques surmontant des cornes de consécration, étaient peut-être rattachés à des larnakès ${ }^{106}$. Le sphinx apparaît sur au moins cinq larnakès de Tanagra ${ }^{107}$. Aucune larnax crétoise ne comporte une représentation de sphinx à buste humain. En revanche, un sceau gravé d'un sphinx ailé provient de la tombe 4 d'Haghia Triada, en Crète, où se trouvaient le sarcophage en pierre et une autre larnax en terre cuite ${ }^{108}$.

97. Spyropoulos 1970 b, p. 190-197, fig. 15-17 ; Spyropoulos 1969 a, p. 14, pl. 12, $13 \alpha, 14 \beta$.

98. Vermeule 1965 , p. 141 ; Immerwahr 1995, p. 119 , n. 29 ; Benzi 1999, p. 229.

99. Spyropoulos 1973 , p. 21 , pl. $11 \beta$.

100. French 1961, p. 88-89; Vermeule et Karageorghis 1982, p. 221, n' ${ }^{\circ}$ III . 5.1, XI.31, XI. 32.

101. A. Evans, The Prehistoric Tombs of Knossos, Archaeologia, 59, London, 1906, p. 28-29, fig. $26 a-b$; Kanta 1980,

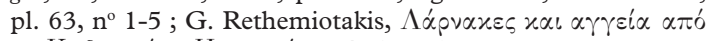

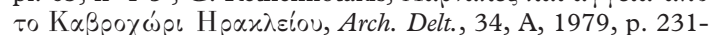
232 fig. 3-6, pl. 93-94; Long 1974, pl. 7, 11, 25, 27, fig. 18, 26, 73, 75 .

102. Spyropoulos 1971 , p. 12 , pl. $18 \beta$; Vermeule 1965 , p. 130, $\mathrm{n}^{\circ} 5$, pl. XXVIII $a$; Dimakopoulou et Konsola 1981, p. $83, \mathrm{n}^{\circ} 11$.

103. Pour une petite figure peinte sur une larnax de Dendron, voir Spyropoulos 1974, p. 12, pl. $10 \alpha$; pour les figures ailées, voir Immerwahr 1995, p. 116.
104. L. Morgan, A Minoan Larnax from Knossos, $A B S A, 82,1987$, p. 171 ; Tzedakis 1971 , p. 221, fig. 9 ;

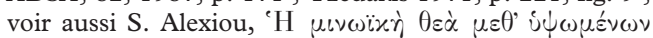

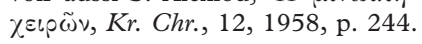

105. Dimakopoulou et Konsola 1981, p. 83 n 11 ; Vermeule 1965, p. 130, pl. XXVIII $a$.

106. Spyropoulos 1969 a, p. 13, pl. $7 \beta$; Spyropoulos 1970 b, p. 188, 196 ; Spyropoulos 1971, p. 8-9.

107. Spyropoulos 1973 , p. 21 , pl. $11 \alpha$, et Spyropoulos 1974 , p. 14 , pl. $10 \beta, 11 \alpha$; Spyropoulos $1970 a$, p. 35, et Dimakopoulou et Konsola 1981, p. 83, $\mathrm{n}^{\circ} 6$; Spyropoulos 1979 , p. 30, 33-35, pl. $20 \beta$; Vermeule 1979 , p. 205, fig. 1.

108. A. Dessenne, Le Sphinx, Étude iconographique, I, Des origines à la fin du second millénaire, Paris, De Boccard, 1957, p. 132-133, $\mathrm{n}^{\circ} 296$, pl. 24 ; Long 1974, p. 13 ; CMS, II. 3, p. 139, n 118 (HM 339). 
En Grèce continentale, dans la grande tombe de Mégalo Kastelli, les sphinx qui ornent une pyxide en ivoire témoignent de son importance dans le monde mycénien ${ }^{109}$. Aux périodes historiques, il tient un rôle essentiel dans les mythes thébains.

Les oiseaux géants et les sphinx sont souvent compris comme protecteurs du défunt, les premiers l'accompagnant dans son dernier périple vers l'au-delà, les seconds gardant la tombe ${ }^{110}$. Ces interprétations développées en regard des témoignages des périodes historiques restent les plus exposées aux critiques. Les seuls textes mycéniens se rapportant à la religion sont des listes d'offrandes. De ce fait, on ne peut prêter avec certitude une finalité eschatologique aux êtres fantastiques.

Il en va de même pour la lecture de la scène comprenant un bateau sur la larnax de la tombe 47 de Dendron ${ }^{111}$. On peut également s'interroger sur la signification du bateau peint sur une larnax de Gazi en Crète ${ }^{112}$. La présence d'embarcation en contexte funéraire est souvent interprétée comme une allégorie. L'au-delà se situait aux confins du monde et n'était accessible que par un long voyage à travers les eaux, croyance évoquée chez Homère ou chez Hésiode ${ }^{113}$.

\section{CONCLUSION}

À l'image de ce qui se fait en Crète, l'usage de contenants funéraires est attesté en Grèce continentale où des inhumations en contenants funéraires sont recensées dans onze cimetières. Ils sont le plus souvent en terre cuite et datés de l'HR III, comme dans les deux cimetières de Tanagra. Cependant, une étude de la position des restes osseux a mis en évidence la présence, jusque-là insoupçonnée, de contenants en matière périssable dans les cimetières d'Éleusis et de Pérati. Dans l'état actuel des recherches, la présence d'inhumations en contenants dans les autres cimetières de Grèce continentale demeure sporadique.

Inhumations en contenants et inhumations déposées sur le sol coexistent dans un même cimetière, parfois dans une même tombe. À Dendron et Géphyra, l'inhumation en contenant n'est pas pratiquée pour toute la population, mais pour une minorité d'individus (voir $\$ 2.1$ ). À Prosymna, une larnax en terre cuite et des brancards en bois ont été découverts dans quatre tombes différentes. En revanche, la coexistence de larnakès en terre cuite et de contenants en bois ne semble pas attestée dans un même cimetière. Or le bois et l'argile sont également dis-

109. Spyropoulos 1972 a, p. 310, pl. $252 \alpha$; Dimakopoulou et Konsola 1981 , p. 55, pl. 26.

110. Pour le domaine des croyances, voir M. P. Nilsson, The Minoan-Mycenaean Religion and its Survival in Greek Religion, Skrifter, IX, Lund, C. W. R. Gleerup, 1950, p. 626 ; pour les sphinx, voir E. T. Vermeule, Aspects of Death in Early Greek Art and Pottery, Berkeley, University of California Press, 1979, p. 69, 171
111. Spyropoulos 1973, p. 21, pl. $10 \propto$.

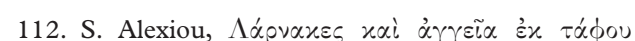

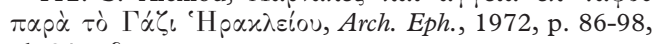
pl. $34 \alpha-\beta$.

113. Odyssée, IV, v. 563-568; Hésiode, Les travaux et les jours, v. 170-173. 
ponibles en Crète et en Grèce continentale : le choix du matériau ne résulte donc pas d'une contrainte naturelle, mais relève à l'évidence d'un choix culturel.

Par ailleurs, la richesse du mobilier ne dépend pas du mode d'inhumation adopté. Des armes proviennent de la tombe 8 de Dendra, qui contenait notamment une inhumation en cercueil $^{114}$. Dans les tombes d'Éleusis et de Pérati, le mobilier associé aux inhumations en contenants s'avère modeste, majoritairement constitué de céramiques ${ }^{115}$. À Dendron et Géphyra, certaines tombes renfermant des larnakès étaient riches en mobilier, d'autres dépourvues de matériel. Les dépôts d'objets et la présence de larnakès dans les tombes ne peuvent donc servir d'argument pour désigner le statut d'un défunt. L'emploi de larnakès et de cercueils semble ainsi motivé par un autre facteur. Le défunt déposé dans un contenant funéraire paraît tout au plus individualisé. Cependant, peut-on suggérer, comme P. Muhly, que des individus appartenant à un "large spectre socio-économique " étaient inhumés dans des contenants ou sur des brancards ${ }^{116}$ ?

R. Hägg et F. Sieurin se sont penchés sur les origines des cercueils en bois au Bronze Récent ${ }^{117}$. Dans leur étude, les traces de bois ont été attribuées de préférence à des cercueils, de sorte que l'existence des brancards s'en est trouvée sous-estimée. En supposant qu'un cercueil en bois se trouvait dans la tombe III du Cercle A de Mycènes, il s'agit du seul contenant en bois qui précéderait les premières inhumations en cercueils du MR IB / II dans la région de $\mathrm{Cnossos}^{118}$. En revanche, les inhumations en cercueils identifiées à Éleusis, Athènes et Dendra sont contemporaines des exemples crétois et précèdent les premières larnakès en terre cuite de Tanagra.

L'usage de prototypes en bois dans la fabrication des larnakès en forme de coffre à l'HR III ne peut être réfuté. Les parois des larnakès sont constituées de panneaux et de pieds modelés dans le prolongement des montants, à l'instar de coffres en bois formés de planches ${ }^{119}$. En revanche, les contenants en bois oblongs plus anciens que les larnakès de l'HR III n'ont probablement pas influencé l'élaboration des larnakès en terre cuite. Notons que les cercueils oblongs renfermaient des défunts en position allongée.

La documentation très lacunaire rend impossible l'établissement d'un parallèle probant entre la position originelle des défunts à l'intérieur des larnakès crétoises et à l'intérieur de celles des cimetières de Tanagra. En effet, notre étude a démontré que la position des membres inférieurs à l'intérieur des larnakès en bois d'Éleusis et de Pérati ne se retrouve pas en Crète. Dans ces deux cimetières, une seule position est observée : les genoux sont fléchis au-dessus du fond du contenant, l'un d'eux prenant appui contre l'un des grands côtés de la larnax $^{120}$.

114. Persson 1942 , p. 41, 43-45.

115. Mylonas 1975, vol. B, p. 142, 192 ; Iakovidis 1969, p. 193.

116. Muhly 1996, p. 208.

117. Hägg et Sieurin 1982 , p. 177-186, table I.

118. Katsamba, Tombes $2-5$ et 7 : S. Alexiou, ' $T \sigma \tau \varepsilon \rho o-$

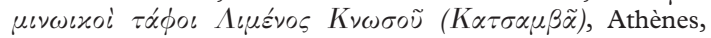
Archaiologiki Etaireia, 1967, p. 3, 41 ; Haghios Ioannis,
Tombe dite de guerrier: M. S. F. Hood, Another Warrior-Grave at Ayios Ioannis near Knossos, $A B S A$, 51, 1956, p. 84 ; voir Muhly 1996, p. 210.

119. Rutkowski 1968, p. 223.

120. En revanche, en Crète, on distingue trois types de position selon le degré de fléchissement des genoux et la disposition des membres inférieurs dans le contenant. 
Dans les cimetières de Grèce continentale, le mobilier des tombes qui renfermaient des contenants funéraires est typiquement mycénien. La découverte d'ossements de chèvres et de coupes ne se différencie pas des trouvailles signalées dans les tombes où se trouvaient des inhumations déposées sur le sol. Les rites funéraires incluant repas et boisson devaient être répandus dans les cimetières mycéniens et ne dépendaient pas du mode d'inhumation. En observant les scènes funéraires dépeintes sur les larnakès, nous ne pouvons toutefois affirmer si le même cérémonial était pratiqué lors de chaque inhumation.

Par conséquent, la coexistence de différents types d'inhumation dans les cimetières mycéniens reflète davantage un choix individuel d'inhumation qu'une conception différente de la mort. Cependant, on ne peut sous-estimer dans l'usage de larnakès en terre cuite la forte affinité avec la tradition funéraire crétoise. L'usage de contenants funéraires demeure une tradition caractéristique de la Crète. À l'exception des enfants inhumés en pithoi, c'est en Crète et non en Grèce continentale que les inhumations en contenants funéraires sont le plus souvent attestées. Au MR III, la présence d'ateliers est indiquée par A. Kanta dans les régions de Rethymnon et d'Arménoi ${ }^{121}$. Les échanges entre la Grèce continentale et la Crète ont certainement favorisé la circulation d'un savoir-faire via les artisans ou les commerçants.

Laetitia PHIAlon, Sandrine FARrugio, Maison René-Ginouvès de l'Archéologie et de l'Ethnologie, UMR 7041 du CNRS, 21, allée de l'Université, 92023 Nanterre Cedex.

\title{
RÉFÉRENCES BIBLIOGRAPHIQUES (POUR LES DEUX TABLEAUX)
}

\author{
Athènes Immerwahr 1971, p. 101, pl. 90, 99. \\ Dendra Persson 1942, p. 41, 43-45. \\ Éleusis Mylonas 1975, vol. A, p. 195-198, 307-311, fig. 76 ; vol. B, p. 10-24, 139- \\ 146, 190-194, 198-201, fig. 95, 98, 120, 132 ; vol. Г, pl. 34-35, 66, 97, \\ $106,153,185,189$. \\ H. Kosmas Mylonas 1959, p. 53, 61-62, dessin 14. \\ Ktouri Béquignon 1932, p. 148-149, fig. 41. \\ Mycènes, tombe Wace 1932, p. 8-9, fig. 4. \\ Mycènes, Porte Wace 1921/1923, p. 36, fig. 4, pl. VI C. \\ Pérati Iakovidis 1969 , p. 192, fig. 51, pl. $179 \beta$. \\ Prosymna Blegen 1937, p. 53-56, 456, fig. 99, 101, $\mathrm{n}^{\circ} 1206$; pour la datation, voir \\ Shelton 1996, p. 37.
}

121. Kanta 1980, p. 290-293. Les larnakès en terre cuite de type en coffre sont très répandues en Crète, principalement au centre et à l'Ouest. Pour les larnakès en forme de baignoire, voir M. Tsipopoulou, L. Vagnetti, Attributions for Some Late Minoan III East Cretan Larnakes, dans
R. Laffineur, Ph. Betancourt éd., TEXNH, Craftsmen, Craftswomen and Craftsmanship in the Aegean Bronze Age (Aegaeum, 16), Liège, Université de Liège, 1997, p. 473479, pl. CLXXX-CLXXXVIII. 
Tanagra, D.

Tanagra, G.

Tanagra, ? Thèbes, Ism. Thèbes, M. K. Vraserka
Spyropoulos 1970 a, p. 31, 33-36, fig. 1, pl. $48 \alpha-\beta, 49$; Spyropoulos 1973, p. 14-15, 17, 21, fig. 1, pl. $10 \alpha-\beta, 11 \alpha-\beta$; Spyropoulos 1974, p. 10, 14, pl. $10 \beta, 11 \alpha-\beta$; Spyropoulos 1976, p. 61 ; Spyropoulos 1977, p. 27, 31, pl. $12 \alpha-\beta, 13 \alpha$; Spyropoulos 1979, p. 30, 33-35, pl. $20 \alpha-\beta, 21 \alpha-\beta$.

Dimakopoulou et Konsola 1981, p. 83 ; Spyropoulos 1969 b, p. 22-24 ; Spyropoulos 1970 b, p. 184-197, fig. 4-17 ; Spyropoulos 1972 b, p. 206209, pl. 209 B ; Spyropoulos 1969 a, p. 7-12, 14, pl. 1-3, $4 \alpha-\beta, 5 \alpha-\beta$, $6 \alpha-\beta, 7 \alpha, 12,13 \alpha, 14 \beta$; Spyropoulos 1971, p. 8, 12-13, pl. $7 \alpha-\beta, 8 \alpha-\beta$, $9 \alpha, 10 \alpha, 17 \alpha-\beta, 18 \alpha-\beta, 19 \alpha-\beta$.

Dimakopoulou et Konsola 1981, p. 82-83.

Keramopoullos 1917, p. 92, fig. 66.

Spyropoulos 1972 a, p. 309.

Dimakopoulou 1987, p. 69-78, fig. 1, pl. 1-10.

\section{ABRÉVIATIONS BIBLIOGRAPHIQUES}

Baxevani 1995

Benzi 1999

Béquignon 1932

Blegen 1937

Cavanagh et Mee 1995

CMS

Dimakopoulou

et Konsola 1981

Dimakopoulou 1987

Duday 1995

French 1961

Hägg et Sieurin 1982

Iakovidis 1969
K. Baxevani, A Minoan Larnax from Pigi Rethymou with Religious and Funerary Iconography, dans C. Morris éd., Klados, Essays in Honour of f. N. Coldstream, Londres, University of London, p. 15-33.

M. Benzi, Riti di passagio sulla larnax dalla Tomba 22 di Tanagra ?, dans

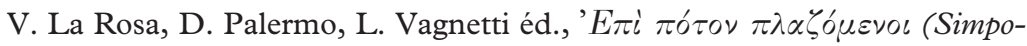
sio italiano di Studi Egei dedicato a Luigi Bernabo Brea e Giovanni Pugliese Carratelli, Roma, 1998), Rome, Scuola archeologica italiana di Atene, p. 215-233.

Y. Béquignon, Études thessaliennes, $B C H, 56$, p. 89-191.

C. W. Blegen, Prosymna, The Helladic Settlement preceding the Argive Heraion, Cambridge, University Press.

W. Cavanagh, C. Mee, Mourning before and after the Dark Age, dans C. Morris éd., Klados, Essays in Honour of $\mathcal{F}$. N. Coldstream, Londres, University of London, p. 45-61.

Corpus der minoischen und mykenischen Siegel.

K. Dimakopoulou, D. Konsola, Musée archéologique de Thèbes, Athènes, Caisse des recettes archéologiques (TAPA).

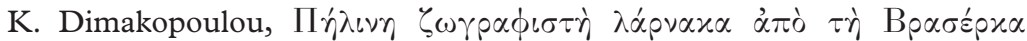
'Apro $i \delta \alpha \varsigma$, dans L. Kastrinaki, G. Orphanou, N. Giannadakis éd.,

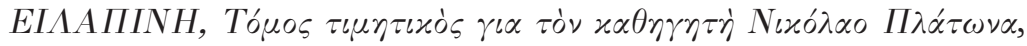
Héraclion, Dèmos Hèrakleiou, p. 69-78.

H. Duday, Anthropologie "de terrain ", archéologie de la mort, dans La Mort, passé, présent, conditionnel (La Roche-sur-Yon, 1994), La Roche-surYon, Groupe vendéen d'Études préhistoriques, p. 33-58.

E. French, A Chariot Larnax from Mycenae, $A B S A$, 56, p. 88-89.

R. Hägg, F. Sieurin, On the Origin of the Wooden Coffin in Late Bronze Age Greece, $A B S A, 77$, p. 177-186.

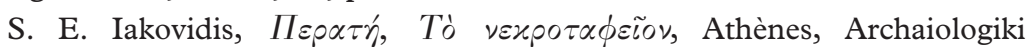
Étaireia. 
Immerwahr 1971

Immerwahr 1995

Kanta 1980

Keramopoullos 1917

Kontorli-

Papadopoulou 1996

Long 1974

Mavriyannaki 1972

Mountjoy 1999

Muhly 1996

Mylonas 1959

Mylonas 1975

Persson 1942

Rutkowski 1968

Spyropoulos 1969 a

Spyropoulos $1969 b$

Spyropoulos $1970 a$

Spyropoulos $1970 b$

Spyropoulos 1971

Spyropoulos 1972 a

Spyropoulos $1972 b$

Spyropoulos 1973

Spyropoulos 1974

Spyropoulos 1975

Spyropoulos 1976
S. A. Immerwahr, The Athenian Agora, The Neolithic and Bronze Ages (The Athenian Agora, XIII), Princeton, ASCA.

S. A. Immerwahr, Death and the Tanagra Larnakes, dans J. Carter, S. Morris éd., The Ages of Homer, A Tribute to E. Townsend Vermeule, Austin, University of Texas Press, p. 109-121.

A. Kanta, The Late Minoan III Period in Crete, A Survey of Sites, Pottery and their Distribution, SIMA, 58, Göteborg, P. Åström.

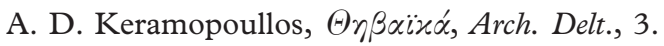

L. Kontorli-Papadopoulou, Aegean Frescoes of Religious Character (SIMA, 117), Göteborg, P. Åström.

R. Long, The Ayia Triadha Sarcophagus, A Study of Late Minoan and Mycenaean Funerary Practices and Beliefs (SIMA, 41), Göteborg, P. Åström.

C. Mavriyannaki, Recherches sur les larnakès de Crète Occidentale (Incunabula Graeca, 54), Rome, Éd. dell'Ateneo.

P. A. Mountjoy, Regional Mycenaean Decorated Pottery, II, Rahden/Westf., Verlag Marie Leidorf.

P. Muhly, Furniture from the Shaft Graves: the Occurrence of Wood in Aegean Burials of the Bronze Age, $A B S A$, 91, p. 197-211.

G. E. Mylonas, Aghios Kosmas: An Early Bronze Age Settlement and Cemetery in Attica, Princeton University Press.

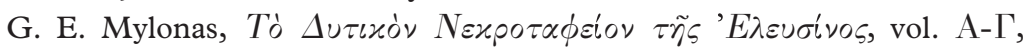
Athènes, Archaiologiki Étaireia.

A. W. Persson, New Tombs at Dendra, Lund, C. W. R. Gleerup.

B. Rutkowski, The Origin of the Minoan Coffin, $A B S A, 63$, p. 219-227.

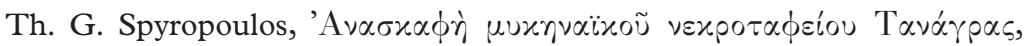
Praktika, p. 5-15.

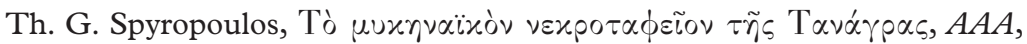
II, p. 20-25.

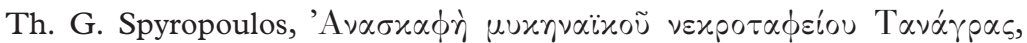
Praktika, p. 29-36.

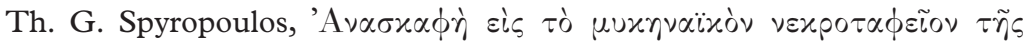

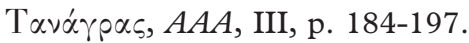

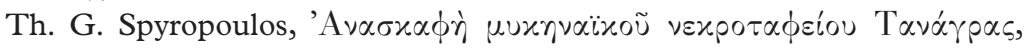
Praktika, p. 7-14.

Th. G. Spyropoulos, Megalo Kastelli, Arch. Delt., 27, B2, p. 309-312.

Th. G. Spyropoulos, Terracotta sarcophagi, Archaeology, 25, 1972, p. 206-209.

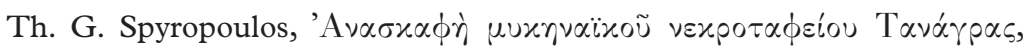
Praktika, p. 11-21.

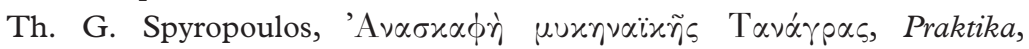
p. 9-33.

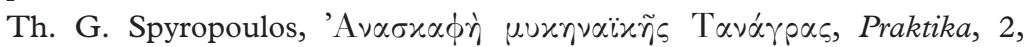
p. 415-427.

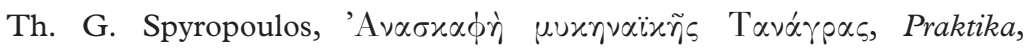
p. 61-68. 
Spyropoulos 1977

Spyropoulos 1979

Spyropoulos 1984

Tzedakis 1971

Vermeule 1965

Vermeule 1979

Vermeule

et Karageorghis 1982

Vikatou 2001

Wace 1921-1923

Wace 1932

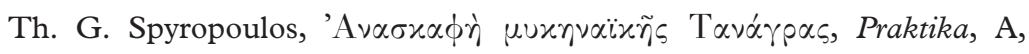
p. 25-31.

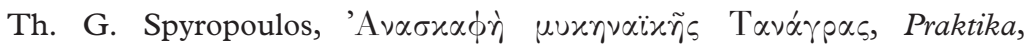
p. 27-36.

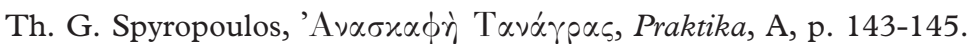

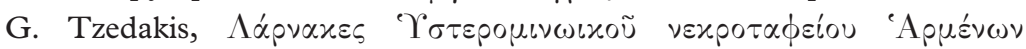

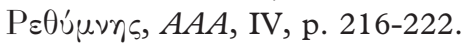

E. T. Vermeule, Painted Mycenaean Larnakes, $\mathcal{F H S}$, 85, p. 123-148.

E. T. Vermeule, A Painted Mycenaean Coffin, dans E. Berger, R. Lullies éd., Antike Kunstwerke aus der Sammlung Ludwig, Bâle, Archäologischer Verlag, p. 201-205.

E. T. Vermeule, V. Karageorghis, Mycenaean Pictorial Vase Painting, Londres, Harvard University Press.

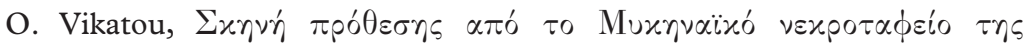

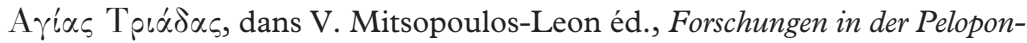
nes (Akten des Symposions anlässlich der Feier " 100 fahre Österreichisches Institut Athen ", Athen, 1998), Athènes, Österreichisches Archäologisches Institut, p. 273-284.

A. Wace, Mycenae, The Lion Gate, $A B S A, 25$, p. 9-38.

A. J. B. Wace, Chamber Tombs at Mycena (Archaeologica, 82), Oxford, The Society of Antiquaries. 